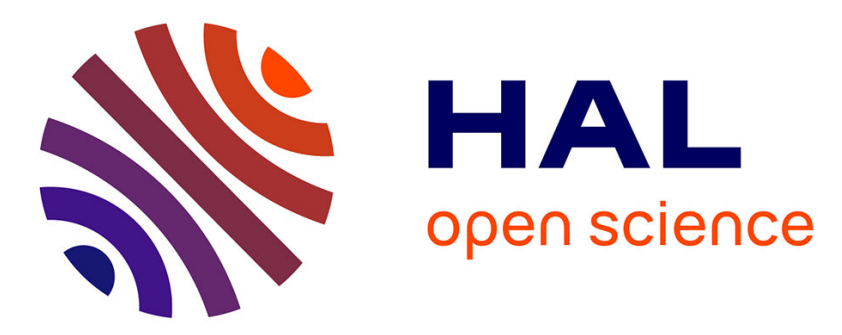

\title{
Pratiques pastorales et stratégies foncières en vallée d'Oô (Pyrénées centrales)
}

\author{
Gerard Balent, Monique Barrue-Pastor
}

\section{To cite this version:}

Gerard Balent, Monique Barrue-Pastor. Pratiques pastorales et stratégies foncières en vallée d'Oô (Pyrénées centrales). Revue Géographique des Pyrénées et du Sud-Ouest, 1986, L'élément et le système, 57 (3), pp.403-447. 10.3406/rgpso.1986.4959 . hal-02865890

\section{HAL Id: hal-02865890 \\ https://hal.science/hal-02865890}

Submitted on 12 Jun 2020

HAL is a multi-disciplinary open access archive for the deposit and dissemination of scientific research documents, whether they are published or not. The documents may come from teaching and research institutions in France or abroad, or from public or private research centers.
L'archive ouverte pluridisciplinaire HAL, est destinée au dépôt et à la diffusion de documents scientifiques de niveau recherche, publiés ou non, émanant des établissements d'enseignement et de recherche français ou étrangers, des laboratoires publics ou privés. 


\title{
Pratiques pastorales et stratégies foncières en vallée d'Oô
} (Pyrénées centrales)

\author{
G. Balent, Monique Barrué-Pastor
}

Citer ce document / Cite this document :

Balent G., Barrué-Pastor Monique. Pratiques pastorales et stratégies foncières en vallée d'Oô (Pyrénées centrales). In: Revue géographique des Pyrénées et du Sud-Ouest, tome 57, fascicule 3, 1986. L'élément et le système. pp. 403-447;

doi : https://doi.org/10.3406/rgpso.1986.4959

https://www.persee.fr/doc/rgpso_0035-3221_1986_num_57_3_4959

Fichier pdf généré le 06/04/2018 


\title{
Résumé
}

Au cours du siècle écoulé, les activités agricoles en montagne ont connu une régression très importante. Dans les Pyrénées centrales, les strictes réglementations communautaires visant l'usage du territoire pastoral se sont relâchées. La diversification sociale des agriculteurs, le blocage du marché foncier, la diminution des troupeaux, et le développement de pratiques pastorales individuelles, ont entraîné une baisse générale de la qualité des parcours et de la fertilité des sols qui pose le problème de la reproductibilité des ressources pastorales. Après une analyse des processus de déprise, dans leurs déterminants agro-écologiques et sociaux, sont envisagées les possibilités d'améliorer la gestion du territoire pastoral et d'assurer la pérennité de ses ressources dans une perspective d'une relance des activités agricoles en montagne.

\section{Resumen}

Pastoreo y estrategia de propiedad : claves del proceso de decadencia de la ganadería de montaña. Durante el pasado siglo, las actividades agrícolas de montaña experimentaron un notable retroceso. En el Pirineo Central, las reglamentaciones comunitarias muy estrictas que tendían a regular el aprovechamiento de los pastizales perdieron eficacia. La diversificación social de los agricultores, el inmovilismo del mercado de la tierra, la disminución global de la cabana así como el desarrollo de un pastoreo individual acarrearon una disminución de la calidad de los recorridos y de la fertilidad de los suelos, planteando la cuestión de la reposición de los recursos en pastos. Análisis del proceso actual de abandono con sus condicionantes agro-ecológicos y sociales. Perspectivas de mejora de la gestión de los pastizales y de conservación de sus .ecursos dentro del marco de una reactivación de la agricultura de montaña.

\begin{abstract}
Pastoral practices and land strategies : two key elements of the decline of mountain husbandry in the central Pyrenees. During this century mountain agricultural activity has declined a lot. In the central Pyrénées strict village rules aimed at controlled pastoral uses have strongly decreased. The social diversification of farmers, the block on the land market, the drop in number of animals as well as the development of individual pastoral practices have produced a drop in the quality of pasture and soil fertility which poses the problem of the reproduction of natural pastoral resources. After an analysis of the process of decline, and some speculation about agro-ecological and social factors, the authors stress the necessity of improving the management of such pastoral areas and their resources.
\end{abstract}




\section{Pratiques pastorales et stratégies foncières dans le processus de déprise de l'élevage montagnard en vallée d'Oô (Pyrénées centrales) *} par G. Balent * et Monique Barrué-Pastor * *

La régression de l'agriculture de montagne et notamment celle des systèmes agro-pastoraux commence à être bien connu (1). Des analyses macro-économiques ont montré que les règles générales de transformation du secteur agraire de notre société, fondées sur un mécanisme d'intégration marchande, de concurrence inter-régionale et d'accumulation différentielle du capital, renforcent les tendances régressives des agricultures montagnardes (Bazin, 1983). Pour préciser la théorie de la régression et tenter de redéfinir les conditions techniques, sociales et économiques d'une agriculture viable et reproductible, nous avons choisi une problématique qui relève des relations entre écosystème et système social. Car répondre à la question du maintien et du développement de l'élevage dans les Pyrénćes, telle qu'elle nous était posée dans le cadre du programme pluridisciplinaire «Elevage pyrénéen » (2) suppose une prise en compte des éléments écologiques, économiques et sociaux impliqués dans cette dynamique. régressive, soit traiter conjointement le double problème de la reproductibilité agronomique et sociale et proposer un système de mise en relation des différents éléments qui participent de ce mécanisme.

Autant peut-on trouver dans les paysages une traduction spatiale des processus d'évolution, autant l'analyse de leurs déterminants exige de pénétrer les rapports qu'une société entretient avec le milieu

(*) Carte hors-texte.

(**) Ingénieur de recherches, URSAD - INRA, Toulouse.

(***) Chargée de recherches, CIMA-UA 366, CNRS, Toulouse.

(1) Notamment par les travaux sur l'Aubrac (R.C.P. Aubrac, 1973-1974), les Vosges (Groupe de Recherches INRA-ENSAA, 1977), le Cause Méjan (BruN et al., 1978), la Margeride (INRA, 1983), les Monts-Dômes (BA7.IN et al., 1983).

(2) Programme pluridisciplinaire de recherche "Elevage Pyrénéen " financé par la DGRST. Groupe de recherches INRA, CNRS, ENSA Toulouse ; 1977-1982. 
exploité. Cette démarche relève à la fois des sciences de la nature et des sciences sociales et repose, d'une manière génćrale, sur la théorie des rentes foncières différentielles (Jollivet, 1978) et sur une con. ception de l'histoire de la nature qui place les sociétés au cœur des dynamiques du milieu naturel (Bertrand, 1975).

La théorie de la rente permet de rendre compte de l'évolution des localisations des productions agricoles à partir des différences de productivité des terres; elle induit "une évaluation économique des potentialités des milieux physiques et biologiques, donc des caractéristiques écologiques " (Jollivet, 1978); elle exprime un rapport social fondé sur le droit de propriété, dont la diversité repose sur une prise en considération de l'autonomie relative des systèmes locaux par rapport aux. transformations d'ensemble, de la place de la propriété foncière dans le système économique. Quant à l'histoire de la nature, et directement en relation avec les premières bases évoquées, nous souscrivons à l'idée que la nature a une histoire liée à l'évolution des rapports de production et de leurs effets sur la nature des sols, leur fertilité et la qualité des ressources récoltées. Ainsi est-ce à la fois en tant qu'objet de l'activité humaine (l'agriculture) et en tant que produit que nous aborderons l'étude des territoires montagnards. Si ces théories permettent de poser les grands principes de la recherche $e_{t}^{t}$ les grandes tendances de transformation des socićtés rurales, elles ne suffisent ni à rendre compte des mécanismes concrets et des transformations des pratiques productives qui alimentent les processus cle régression de certaines zones (Larrère, 1984), ni à comprendre les rapports entre les transformations des sociétés locales et les logiques d'utilisation des ressources naturelles ei apprécier leurs perspectives d'avenir dans leur trajectoire historique.

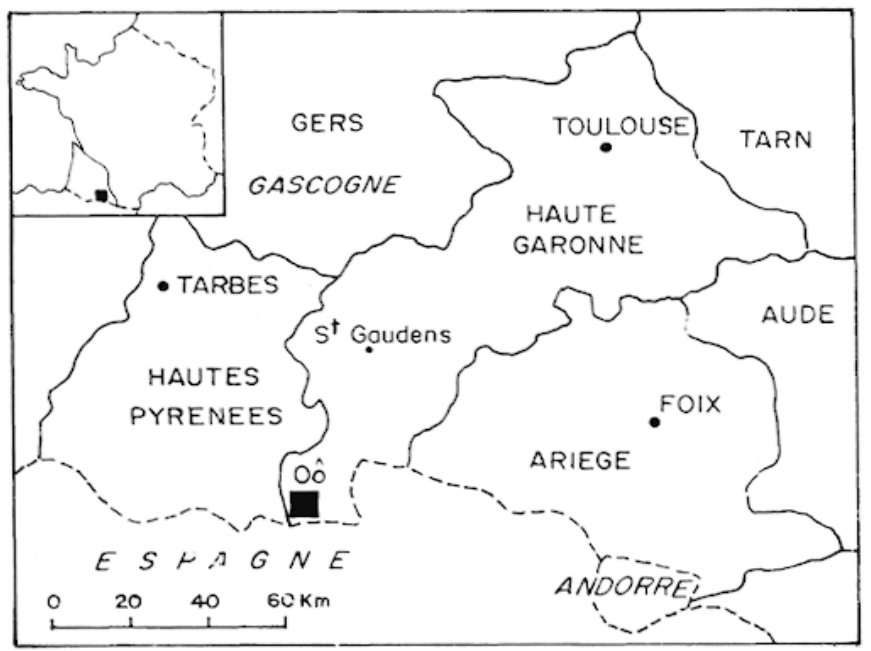

FIG. 1

Localisation de la zone d'étude. 
La zone étudiée se situe dans les Pyrénées centrales qui, comme l'ensemble des montagnes françaises, se caractérisent, depuis un siècle, par un mouvement de régression des activités humaines et de la population, accompagné d'une forte diminution des terres mises en valeur et de la maîtrise de leur gestion. D'une société dont la cohésion était fondée sur un système agricole autarcique et sur l'exploitation maximale des ressources naturelles, on esi passé à une société lucale destructurée sous le poids des nouvelles logiques économiques qui ont entraîné des conflits d'intérêt dans lesquels les agriculteurs ont perdu le contrôle des territoires et du pouvoir local. Dans un contexte d'intégration progressive aux lois dominantes du marché, l'agriculture de montagne s'est trouvée pénalisée par des référents technicoéconomiques issus des zones plus productives et par des règles du marché qui privent ces productions d'une valorisation différenciée. La régression agricole s'est récemment accentuée avec le développement d'un tourisme non maîtrisé comme complémentaire et responsable d'une forte spéculation foncière.

Dans ce contexte historique, et pour analyser les mécanismes de la déprise agricole, un ensemble de recherches coordonnćes portent sur l'évolution du fonctionnement des svstèmes pastoraux, sur la dynamique écologique et agronomique des couverts végétáux, sur l'évolution de l'utilisation du sol, sur la caractérisation des systèmes de pâturage (modalités de pâturage, règlements pastoraux, performances et reproduction des animaux), sur les stratégies foncières et la dynamique sociale.

Nous nous sommes attachés à l'étude de la gestion des ressources pastorales et des ressources fourragères de fond de vallée, qui jouent un rôle fondamental pour l'élevage pvrénéen. Plus que les pâturages communaux d'altitude, elles sont aujourd'hui les enjeux et l'objet des conflits les plus importants, aussi bien des points de vue technique que sociologique. Ces surfaces constituent l'essentiel des richesses territoriales nécessaires au fonctionnement des systèmes d'élevage, qu'il s'agisse de la constitution des réserves en foin ou du pâturage hivernal nécessaire à l'équilibre alimentaire des troupeaux (Gibon, 1981, Balent et Gibon, 1980, 1986). Ce sont aussi celles dont i'appropriation est privée et qui sont l'objet d'enjeux qui traduisent une modification de la structure sociale au détriment des éleveurs en activité (Barrué-Pastor, 1983). "La propriété foncière de l'espace agriccle et ses modes de faire-valoir sont au centre des rapports sociaux des sociétés rurales " (Frémont et al., 1984).

Les surfaces pastorales et fourragères font par ailleurs l'objet d'une couble gestion individuelle et collective. Dans les Pyrénées centrales, en effet, la pratique de la vaine pâture (3) est encore très vivace et

(3) Liberté de pacager sur " tout le fond rural situé dans la commune à l'exception des propriétés closes par des haies ainsi que des prairies artificielles et des terrains plantés d'arbres fruitiers" (Source: cahiers de délibération du Conseil municipal). 


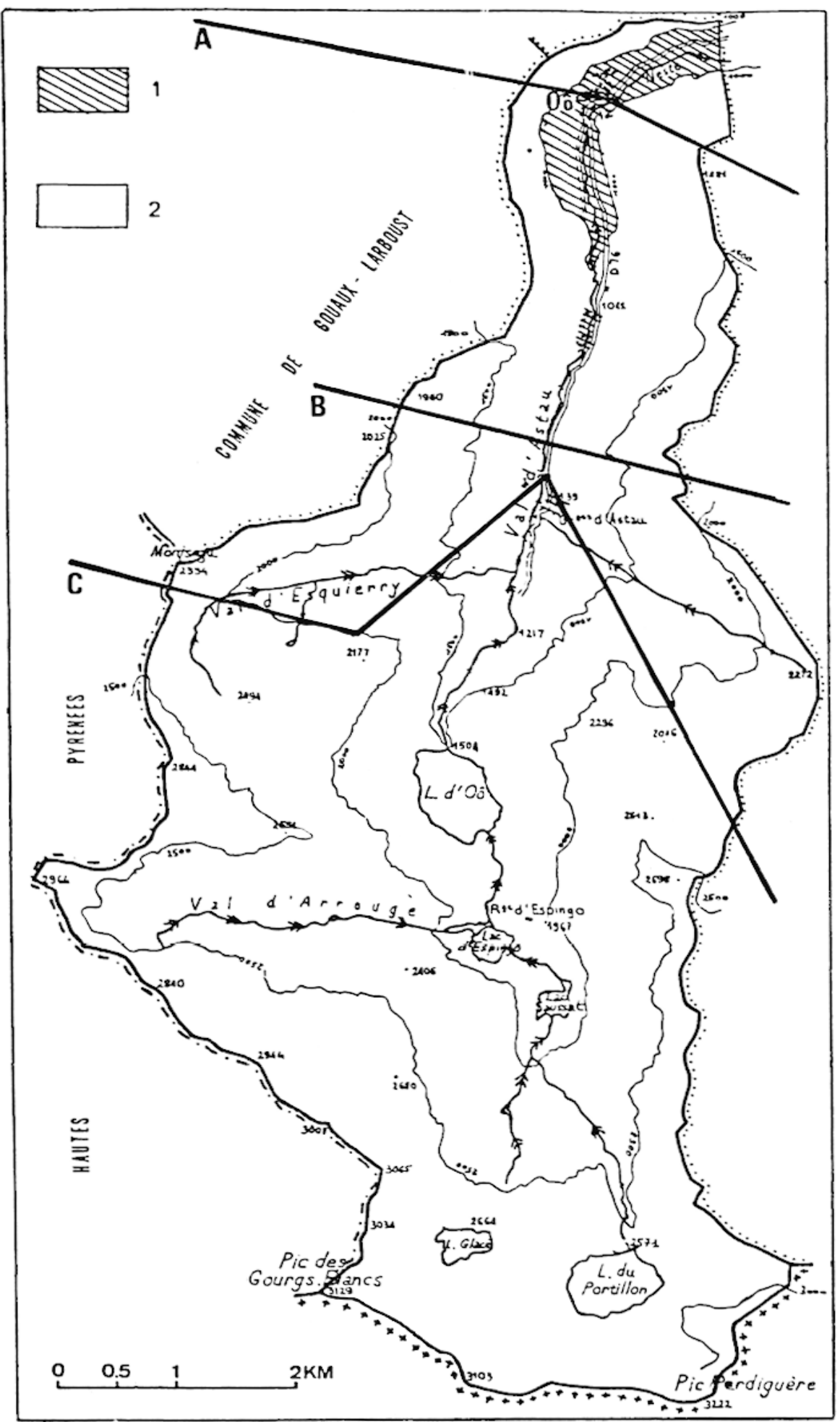

FIG. 2

Propriété foncière en Vallée d'Oô.

1. Propriété privée. - 2. Propriété communale. 


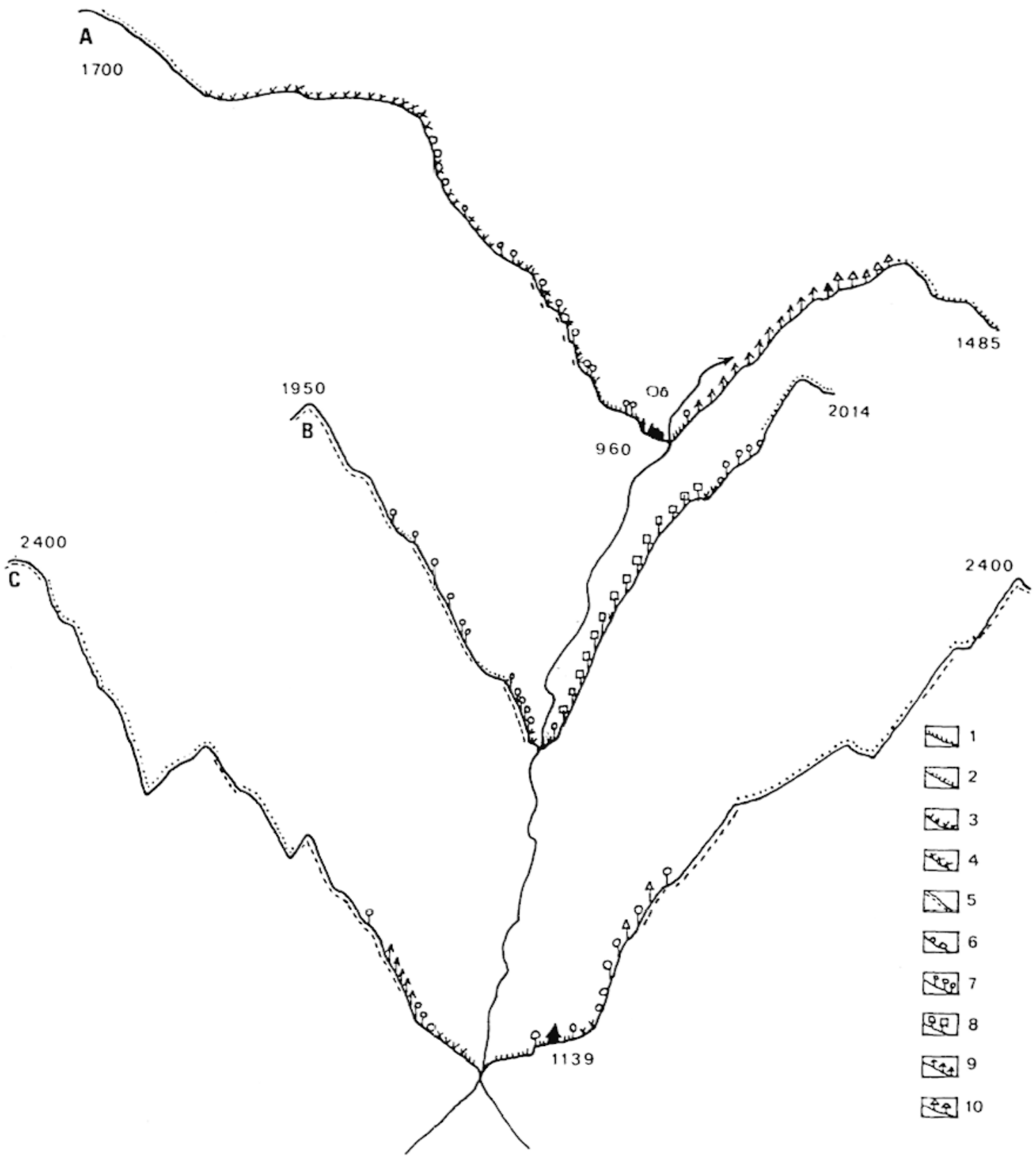

Fig. 3

Profils de végétation en vallée d'Oô selon A, B et C (cf. fig. 2).

1. Pré de fauche. - 2. Pelouse d'estive. - 3. Pâturage. - 4. Pacage embroussaillé. - 5. Affleurement rocheux. - 6. Noisetier. - 7. Feuillus d'enfrichement (hêtre, noisetier, bouleau). - 8. Hêtraie. - 9. Résineux de reboisement RTM (mélèze, sapin). - 10. Sapins.

traduit bien une interpénétration entre deux logiques de gestion. Nous proposons donc l'examen de deux éléments : pratiques pastorales et appropriation foncière, comme deux révélateurs synthétiques et pertinents pour saisir les rapports entre l'évolution des ressources natu- 
relles et de la société montagnarde, soit pour aborder la question de la capacité des agriculteurs à se reproduire socialement et à assurer la reproduction des ressources agronomiques nécessaires à leur fonctionnement. Il s'agit de définir les interactions des différents facteurs de la dynamique des milieux en évitant tout déterminisme social ou naturel.

Quelle signification écologique agronomique et sociale peut-on donner à l'appauvrissement quantitatif et qualitatif des ressources pastorales de fond de vallée ? Comment dater et inscrire ces phénomènes clans une dimension synchronique de façon à pouvoir envisager leurs perspective d'avenir? Autant de questions qui ont justifié une analyse pluridisciplinaire conçue comme une méthode correspondant à un besoin de rationalisation et de compréhension d'une réalité complexe.

Dans l'hypothèse d'une perspective de relance de l'activité d'élevage (4) et dans le contexte local de réinstallation de jeunes agriculteurs, il est urgent de saisir la nature et l'importance du processus actuel de déprise agricole et d'en évaluer les causes et les vitesses d'évolution. Compte tenu des limites du terrain de référence et de la diversité cles situations de montagne, l'intérêt méthodologique de ce travail s'affirme: il alimentera les théories qui soutendent l'analyse des processus régressifs en essayant d'intégrer l'ensemble des paramètres et des déterminants mobilisés dans un schéma de mise en relation plus généralisable qui peut constituer une base de diagnostic, d'analyse, de lancement de nouvelles recherches, mais aussi d'évaluation de la pertinence des politiques d'interventions de l'Etat dans ces zones. A partir de là pourront être envisagés des propositions alternatives de mise en valeur du milieu obéissant à un autre type de logique et de rationalité servant en premier lieu les intérêts de l'agriculture de montagne.

Pour répondre aux exigences de cette démarche complexe nous avons choisi un territoire d'étude limité, mais correspondant à une unité de fonctionnement : la commune est l'entité territoriale pertinente pour l'étude des interactions entre pratiques pastorales et fonctionnement social. Le système pastoral des Pyrénées centrales est en effet caractérisé par le poids des pratiques communautaires liées à la coexistence et aux relations étroites entre propriété privée et propriété collective. "Ce caractère collectif de la vie pastorale est un des caractères fondamentaux de la géographie humaine des Pyrénées, il les oppose fortement à d'autres montagnes françaises " (Chevalier, 1956). Les décisions relatives à la gestion sont prises, dans chaque commune, par le Conseil municipal et portent sur l'ensemble du calendrier de pâturage (vaine pâture hivernale, $\epsilon$ stivale, etc.); à ce niveau, la "collectivité locale " a pris le relais des anciennes "communautés montagnardes ". Si, comme nous le verrons, les logiques

(4) Voir la loi sur la montagne de 1985. 
de fonctionnement et les bases de sa cohésion se sont modifićes, l'unité de gestion des rapports entreterius avec le territoire reste la. commune.

Nos observations portent sur la commune d'Oô, dans la vallée du Larboust (fig. 2), qui appartient au pays du Haut Comminges, à l'extrème sud du département de la Haute-Garonne, en bordure de la frontière d'Espagne; ses 3502 ha en font la plus grande commune du canton, elle constitue à elle seule une entité valléenne, située entre $950 \mathrm{~m}$ et $3222 \mathrm{~m}$. Oó, comme la plupart des communes de cette partie des Pyrénćes dispose de la gamme compète des surfaces pastorales nécessaires au fonctionnement des systèmes d'élevage (fig. 3): les estives (1 100 ha), pâturages communaux d'altitude, utilisés collectivement pendant l'été; les zones interniédiaires (750 ha), pâturages communaux et privés, utilisés collectivement au printemps et à l'automne; les prés de farche (75 ha) et les chumps (10 ha), objet d'une double utilisation, individuclle - (durant la période de récolte des foins et des céréales) - et collective - (vaine pâture hivernale. Les systèmes de production ovin et bovin sont orientés vers la seule production de viande.

La répartition de la propriété foncière traduit l'importance des territoires communaux : $94 \%$ (tabl. 1) et l'exiguïté des structures agraires: une centaine de propriétaires se partagent 197 ha de surface cadastréc, mais la commune ne compte en 1980 que 16 exploitations dont la surface totale utilisée varie de 3 à 16 ha (moyenne des propriétés : 6 ha). Au-delà des chiffres, cette commune présente une grande diversité de types d'exploitants et d'exploitations, non seulement quant aux structures (tabl. 2) mais surtout à propos de projets professionnels et de systèmes de production (Barruć-Pastor, 1980).

La commune d'Oô, bien que marquéa par l'exode et la déprise de l'activité agricole, a conservé un potentiel agricole parmi les plus importants du canton de Bagnères de Luchon. Les 16 exploitations totalisaient en 1982, 145 bovins et 880 ovins adultes, dont respectivement 120 et 450 sont hivernés sur place. L'installation récente de jeunes dans l'agriculture y pose avec une acuité nouvelle la question de l'avenir de l'élevage dans une zone par ailleurs marquée par une relance des activités touristiques et thermales liées à la proximité de Bagnères de Luchon.

\section{D'un système de culture à un système d'élevage : l'utilisation d'une rente de situation}

\section{Evolution des rapports entre élevage et agriculture dans le Haut Comminges au $\mathrm{XIX}^{\prime \prime}$ siècle.}

L'idée de la subordination de l'élevage à l'agriculture vivrière jusqu'au XIX" siècle se retrouve dans la plupart des études récentes sur les Pyrénées centrales. L'importante pression démographique des 
TABLEAU 1

RÉPARTITION dE LA PROPRIÉtÉ FONCIÈRE dANS LA COMMUNE D'Oô (RGA, 1980).

\begin{tabular}{|c|c|c|c|c|c|c|c|}
\hline & & \multirow{2}{*}{$\begin{array}{l}\text { Superficie } \\
\text { tot ale }\end{array}$} & \multicolumn{2}{|c|}{ Propriété communale } & \multicolumn{2}{|c|}{ Propriété privée } & \multirow{2}{*}{$\begin{array}{l}\text { Propriété } \\
\text { domaniale }\end{array}$} \\
\hline & & & tot ale & dont Forêt & tot ale & dont Forêt & \\
\hline \multirow{2}{*}{$\begin{array}{l}\text { Vallée du } \\
\text { Larboust }\end{array}$} & ha & 12983 & 11241 & 1862 & 1718 & 51 & 24 \\
\hline & $\%$ & 100 & 86,6 & - & 13,2 & - & 0.2 \\
\hline \multirow{2}{*}{$\begin{array}{l}\text { dont com- } \\
\text { munc d'Oô }\end{array}$} & ha & 3502 & 3305 & 462 & 197 & 4 & - \\
\hline & $\%$ & 100 & 94,4 & - & 5,6 & - & \\
\hline
\end{tabular}

TABLEaU 2

TYPOLOGIE DES EXPLOITANTS AGRICOLES ET STRUCTURE DES EXPLOITATIONS.

\begin{tabular}{|c|c|c|c|c|c|c|}
\hline & & $\begin{array}{c}\text { Moyens } \\
(1)\end{array}$ & $\begin{array}{c}\text { Int ermé - } \\
\text { diaires } \\
(2 !\end{array}$ & $\begin{array}{c}\text { Margina- } \\
\text { lisés } \\
(3)\end{array}$ & $\begin{array}{c}\text { Double- } \\
\text { Act ifs } \\
(4)\end{array}$ & $\begin{array}{c}\text { Jeunes } \\
\text { en phase } \\
\text { d'inst all at ion } \\
(5)\end{array}$ \\
\hline \multicolumn{2}{|c|}{ Age moyen } & 44 & 59 & 66,5 & 45 & 31 \\
\hline \multirow{2}{*}{ SAU } & tot ale & 13.5 & 8,8 & 5,7 & 5,6 & 7,5 \\
\hline & $\begin{array}{c}\% \text { en } \\
\text { propriété }\end{array}$ & 48 & 77 & 42 & 20 & 30 \\
\hline \multicolumn{2}{|c|}{$\begin{array}{c}\text { U.G.B. } \\
\text { Effectif moyen }\end{array}$} & 48 & 15,2 & 10,7 & 13,6 & 20.5 \\
\hline \multicolumn{2}{|c|}{$\begin{array}{l}\text { Nombre } \\
\text { d'exploit ations }\end{array}$} & 2 & 3 & 4 & 5 & 2 \\
\hline \multirow{3}{*}{$\begin{array}{c}\text { Superficie } \\
\text { des } \\
\text { exploit. }\end{array}$} & 3 a 6 ha & & 1 & 3 & 3 & \\
\hline & 6,1 a 11 ha & & 2 & 1 & 2 & 2 \\
\hline & 11,1 à $16 h a$ & 2 & & & & \\
\hline \multirow{3}{*}{$\begin{array}{c}\text { Systeme } \\
\text { de } \\
\text { productions }\end{array}$} & Ovin & & & & 1 & \\
\hline & Bovin & 1 & 2 & 2 & 2 & 1 \\
\hline & Mixte & 1 & 1 & 2 & 2 & 1 \\
\hline
\end{tabular}

années 1850, dont les effets ne se feront sentir que vingt ans plus tard, contraint les derniers pasteurs à se transformer en agriculteurs. Le système d'alors repose sur l'utilisation maximale des ressources du territoire et de la force de travail disponible. Nombreux sont les historiens qui estiment que l'agriculture de montagne est à son optimum dans les années 1870 (Chevalier, 1956; Weber, 1983). Dans la vallée d'Oô, les superficies en terres labourées sont les plus importantes à cette époque là et le niveau de fertilisation atteint alors son maximum. 
Le souci de maintenir et d'améliorer la fertilité des parcelles cultivées conditionne les pratiques d'ćlevage : "qui a clu foin a du pain", dit le proverbe; le foin nourrit les animaux, qui donnent de l'engrais, qui fait pousser les cultures. Le climat et l'étendue des pâturages communaux permettent d'hiverner un nombre important d'animaux et de disposer de beaucoup de fumier. Aussi, la vallée d'Oó et l'ensemble des vallées du Haut Comminges, pratiquent un assolement tricnnal (seigle-blé; orge-avoine; sarrazin-pomme de terre-pois) sans jachère, qui est une des particularités du système agro-pastoral que l'on trouve dans toutes les Pyrénées centrales et qui les distingue des autres systèmes montagnards. "Les champs sont gorgés de fumier et les cultures se succèdent sans que jamais la terre ne se repose" (J.A.P.H.G., 1861). Dans ce système, "l'image de la brebis utiliss. comme simple "machine à fumer " et celle de la vache comme principal animal de labour sont tout à fait acceptables" (Féral, 1981). Pendant toute la période hors estive, les brebis utilisent de jour les pâturages communaux et, après avoir parcouru des distances parfois considérables, descendent la nuit sur les champs et les prés de fauche pour y être parquées. Il s'ensuit un transfert d'éléments fertilisants des parcours vers les surfaces cultivées (fig. 4). Mais, à cause du faible niveau de fertilité et de production de ces parcours on peut penser, comme cela a été montré dans d'autres situations (Loiseau, 1983), que les exportations dues aux animaux étaicnt en grancle partic compensées par les apports d'éléments minéraux dus à la fixation symbiotique de l'azote par les légumineuses et à la décomposition de la roche mère. Un tel système permet, de maintenir la fertilité des surfaces cultivées et des surfaces pâturées. Notons qu'il n'en va peut-être pas de même pour l'intégrité physique des pâturages (érosion, hydromorphic, etc.).

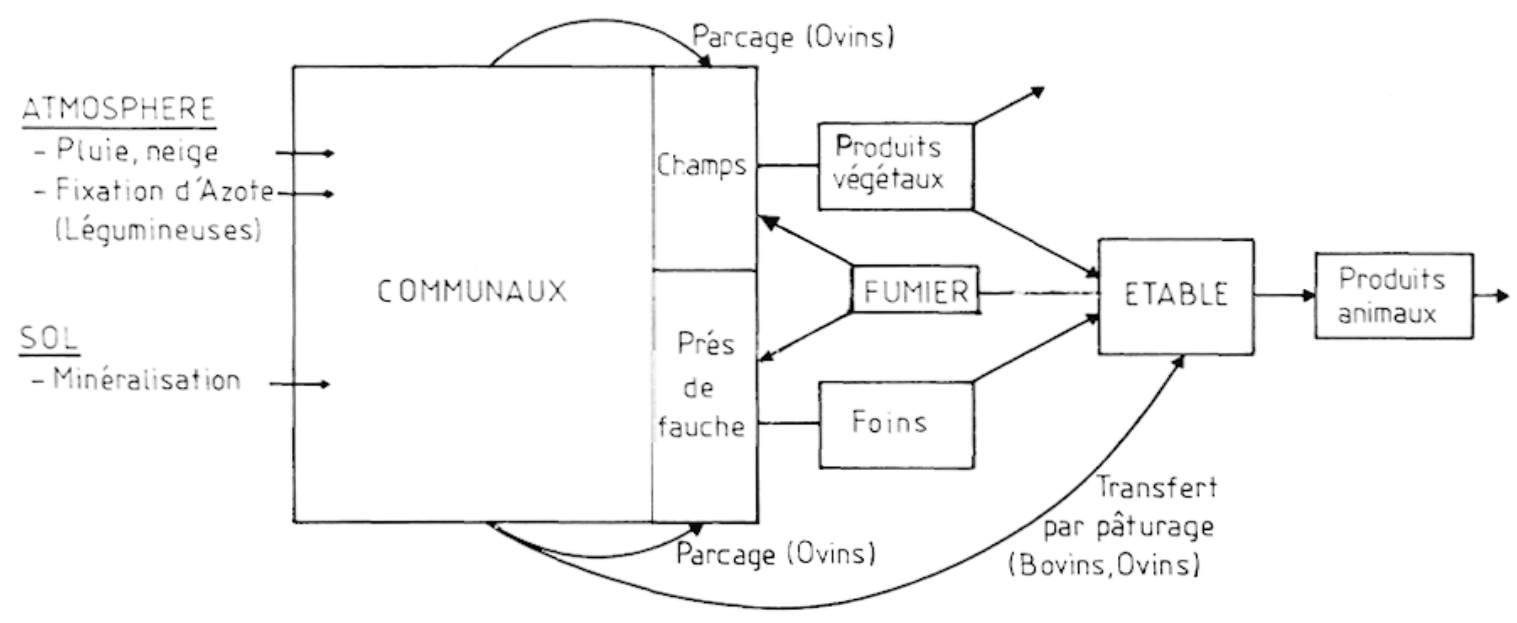

FIG. 4

Gestion des éléments fertilisants dãns le système agro-pastoral des Pyrénées centrales à la fin du XIX $X^{e}$ siècle. 
"Dans ce contexte, le troupeau reste chétif » et non spécialisé. On " tire parti de tout, du lait, de la laine, du croît, du travail " (Féral). Les caractéristiques des races témoignent de ces multiples fonctions. Jusqu'au début du $\mathrm{XX}^{*}$ siècle, la race bovine saint-gironnaise domine largement dans le Haut Comminges, appréciée pour ses qualités de bonne laitière, sa vigueur à la traction, sa résistance à la sous nutrition, son adaptation aux contraintes du séjour en estive, notamment sur sols pierreux et accidentés (Féral). Chez les ovins la situation est plus complexe: les troupeaux sont en général mixtes, composés pour partie d'auroise, de lourdaise (pour la laine) et de castillonaise ces différentes races ont en commun d'être de petit format et d'une grande rusticité. Ces animaux, malgré leur mauvaise conformation viennent compléter les quelques veaux et moutons vendus pour être engraissés en plaine. En effet, quand les propriétaires d'animaux ont obtenu de leur cheptel les éléments nécessaires à la consommation du ménage (céréales, lait, laine) ils lui demandent encore le numéraire dont ils ont besoin pour compléter leur consommation. Ainsi, malgré la logique dominante de subordination de l'élevage à l'agriculture, le bétail représente, par l'argent "frais" que procure la vente de quelques animaux, la seule possibilité d'accumulation du capital.

Sur cette base s'établit la différenciation sociale la plus marquante: des couches paysannes, car elle dépend de l'importance du bien détenu. Ainsi, au cours du premier mouvement de dépopulation de la deuxième moitié du XIX" siècle, seuls ceux qui ont réussi à accumuler du capital privé, soit pour étendre leur territoire cultivé, soit poui élever des animaux, constituer des réserves hivernales ou acquérir des outils, se sont maintenus dans les années 1870 (5).

\section{Evolution socio-économique et utilisation des surfaces de fond de vallée.}

Entre 1850 et 1900 la commune d'Oô enregistre une perte de $40 \%$ de sa population (fig. 5a) alors que la plupart des autres composantes du système agro-pastoral restent stables, à l'exception des outillages et du nombre de bovins (mais les statistiques sur les animaux sont rares et peu fiables). Cette chute démographique entraîne un déve. loppement très rapide de la traction animale : en 1889, chaque " maison " possède déjà une charrue et une paire de bœufs, alors que la première n'a fait son apparition dans le Comminges qu'en 1880; au même moment, le remplacement de la faucille par la faux économisc et allège le travail de récolte.

Avec cet exode, la pression sur le territoire diminue, ainsi que les moyens en main d'œuvre. Les terres labourées commencent à diminuer au début du siècle au profit des surfaces fauchées, évolution qui traduit le passage progressif d'une économie d'autosuffisance à une

(5) On a, pour l'établir, identifé les exploitants à partir du dépouillement des archives municipales. 
économie de marché axée sur les productions animales (augmentation globale des besoins en fourrage accentuce par l'introduction de races plus productives, donc plus exigeantes en nourriture : races gasconne, pour les bovins, et tarasconnaise, pour les ovins). L'abandon de la culture s'accélère ensuite; sur les 92 ha cultivés au début du siècle, 45 seulement le sont encore en 1935 et 10 seulement de nos jours (fig. 5b). Les prés de fauche ne commencent à diminuer de façon significative qu'après les années 1950 (120 ha en 1955, 75 ha en 1980) sous les effets du développement de la mécanisation et de la chute du nombre d'animaux.

Cette évolution conjointe des surfaces cultivées et fauchées se traduit par la marginalisation d'une partie de la SAU. I.'ensemble du territoire en propriété privée (à l'origine, en terres et prés) est utilisć jusqu'en 1925. En 1960, $77 \%$ le sont encore. Aujourd'hui l'ensemble des champs et des prés de fauche ne représente plus que 40 à $4.5 \%$ de la surface en propriété privée. Au total, plus d'une cenlaine d'hectares sont donc devenus des pacages utilisés de manière communau. taire et associés aux territoires communaux. Les parce!le:; abandonnées en priorité sont les plus pentues ou les plus śloignés du village: la pression d'utilisation se concentre ainsi vers le fond de la valléc sur les topographies les plus planes et aux abords inmédiats du villaso (carte h.t.). A l'exception de quelques terroirs à vocation bien affirméc (terrasses en aval du village, pour les champs; fonds de vallée inondable, pour les prés de fauche), l'ensemble des affectations ag̣ricoles du territoire de fond de vallée a été modifié.

\section{Utilisation d'une rente de situation liée à l'histoire culturale des parcelles.}

La diminution du nombre d'animaux, donc des quantité de fumier disponible, a modifié la gestion de la fertilité des parcelles de ce territoire. La mise en relation des pratiques de fertilisation et de J'histoire culturale des parcelles fauchées ou pâturées permet d'examiner la reproductibilité de leur fertilité.

Dans les Pyrénées centrales, la production des prairies de fauche est déterminée par la fumure et le niveau de fertilité phosphopotassique du sol (Balent \& Duru, 1984). Les bilans minéraux de trois parcelles fauchées de fertilités différentes (bonne, moyenne et carencée) montrent que, pour les parcelles les plus fertiles, les apports d'azote, de phosphore et de potassium dus au fumier ne compensent pas les exportations liées à la production réellement obtenue (fig. 6). Obtenir une production supérieure à celle permise par les fumures apportées passe par la mobilisation et l'utilisation par les plantes d'une partie des éléments nutritifs accumulés dans le sol quand la parcelle était un champ et recevait une grande quantité de fumier (40 t./ha/an). Sachant que les prés de fauche étudiés sont d'anciens champs, les trois niveaux de fertilité correspondent à trois durées différentes d'utilisation pour la fauche. Quand un champ est trans- 

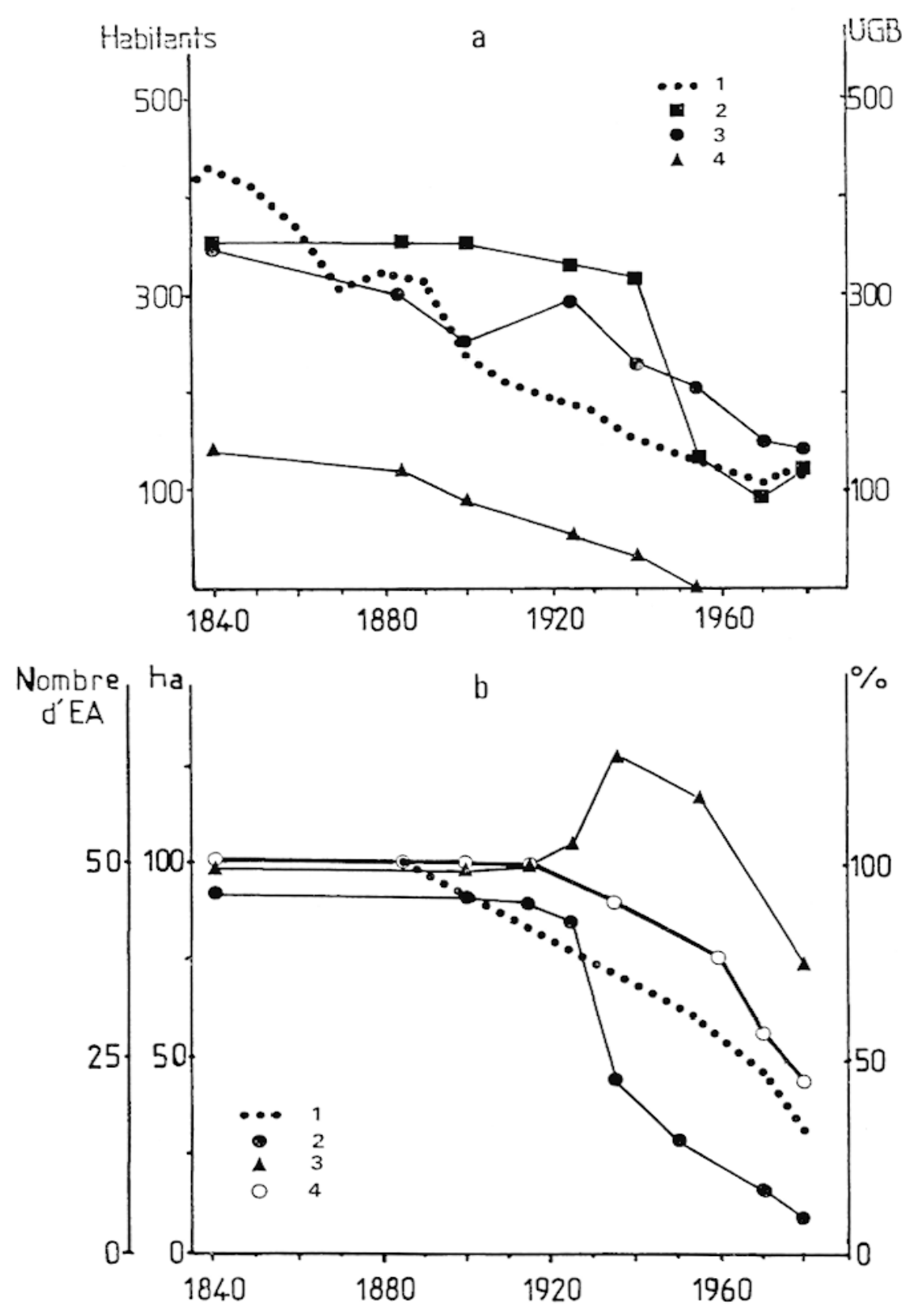

Fig. 5a

Evolution des principales composantes de l'agriculture entre 1840 et 1980.

a. - 1. Population totale. -2 . Ovins. -3 . Bovins. - 4. Equins. - b. - 1. Nombre d'exploitations agricoles. - 2. Champs. - 3. Prés de fauche. - 4. Pourcentage de la surface cadastrée occupée par les champs et les prés de fauche.

formé en pré de fauche, sa productivité annuelle de matière sèche baisse de manière exponentielle pendant vingt à vingt-cinq ans; ensuite la production se stabilise à un niveau en équilibre avec la fumure apportée (fig. 7). Ce "surplus " de matière sèche produite correspond à l'utilisation d'une rente de situation temporaire héritée des modes de mise en valeur antérieurs. 


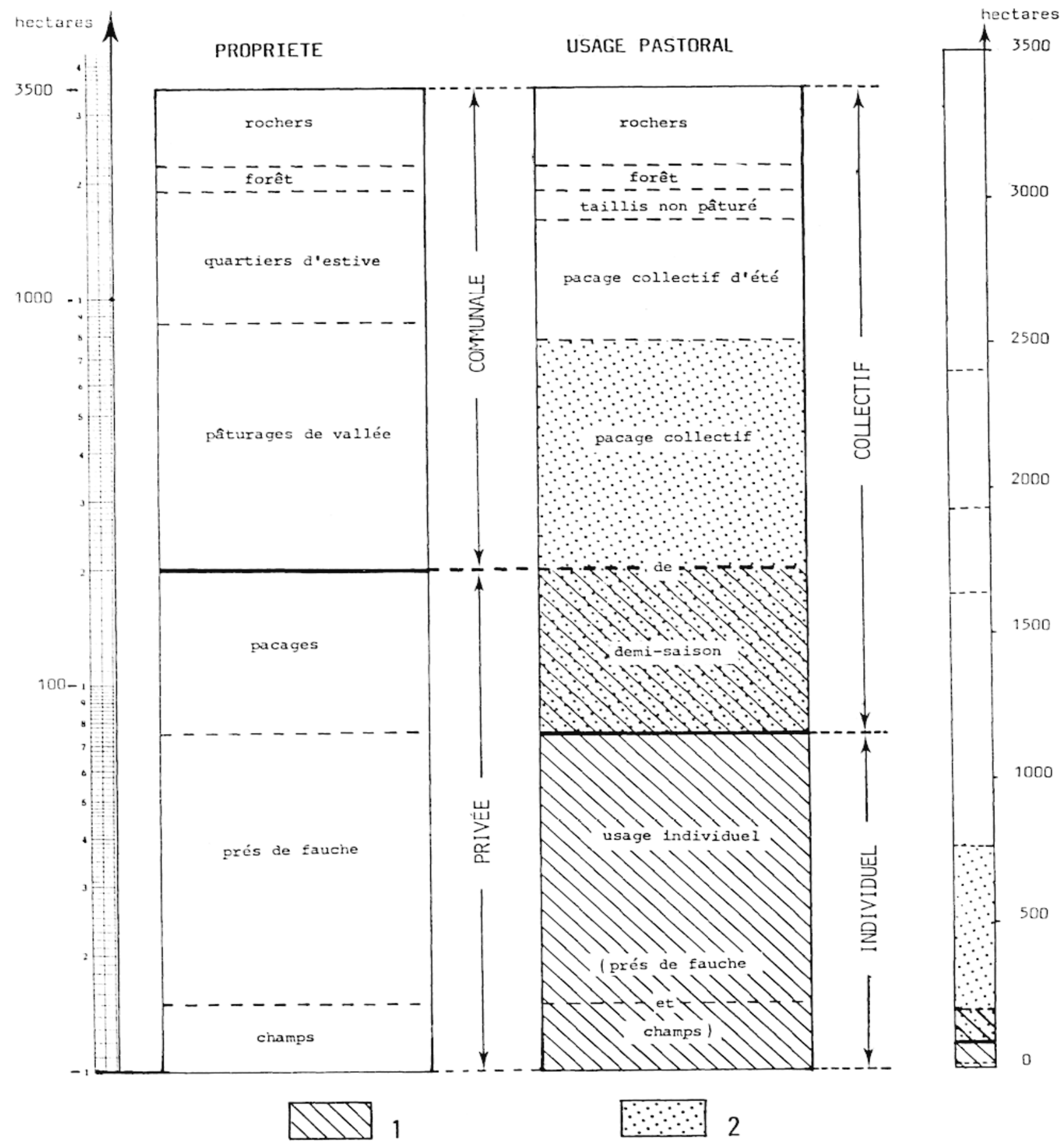

Fig. $5 b$

Propriété foncière et usage pastoral du finage de la commune d'Oô.

1. Vaine pâture hivernale. - 2. Zones intermédiaires.

Les zones uniquement pâturées (anciens prés, anciens champs) ne reçoivent aucune fertilisation. Les animaux, en consomment l'herbe, exportent les éléments fertilisants; leurs déjections n'en restituent 

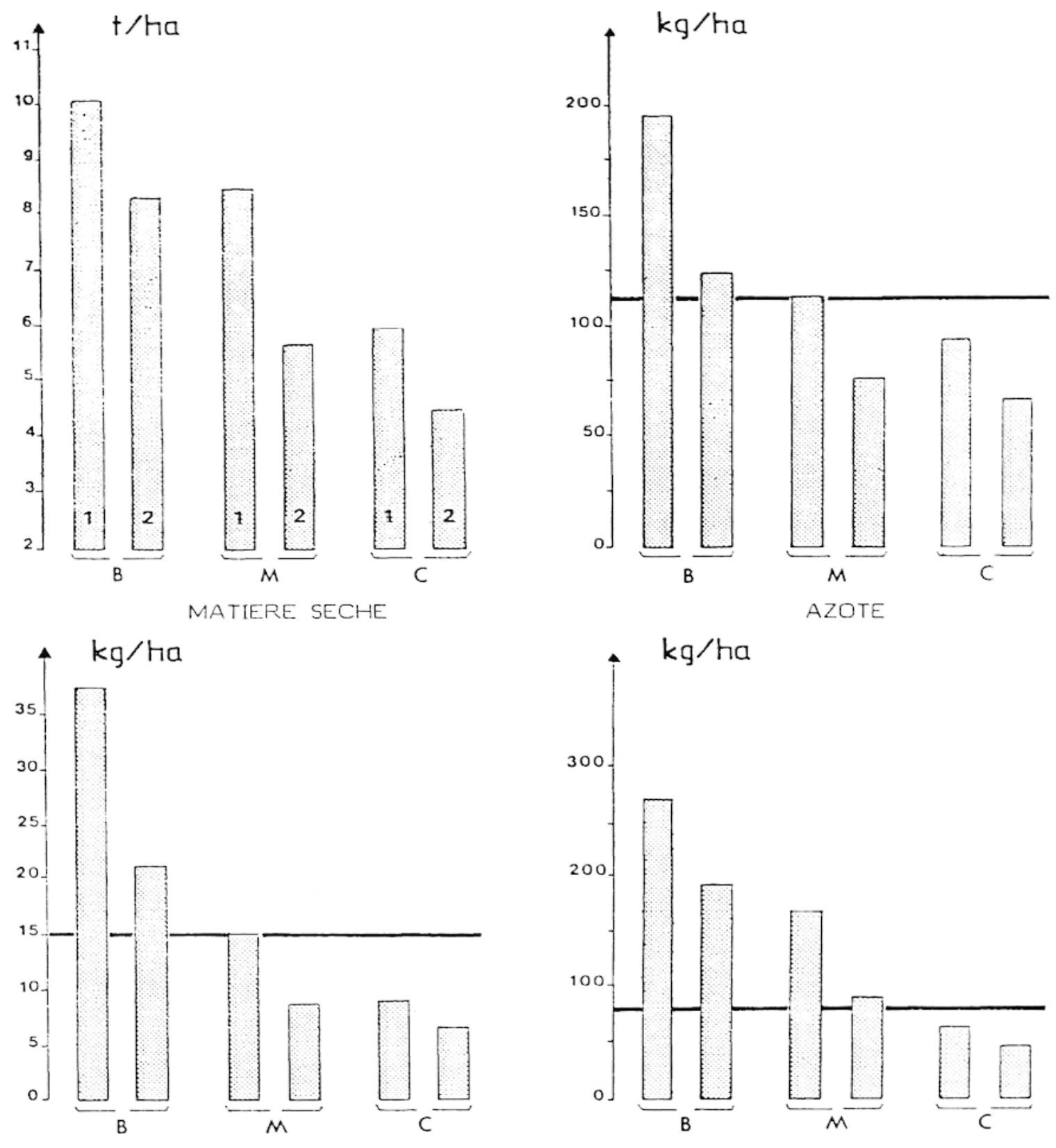

FIG. 6

PHOSPHORE

POTASSIUM

F16. 6

Apports et exportations d'éléments fertilisants sur ies prés de fauche suivant leur exploitation. (d'après Duru et Balent, 1985).

Pour chacun des quatre éléments, trois types de fertilité de parcelle (B : bonne, $M$ : moyenne, C. Carencée), avec (col. 1) ou sans (col. 2) pâturage hivernal. (Le trait horizontal indique le niveau des apports par le fumier).

qqu'une faible partie d'autant moins que, rentrés la nuit, ils laissent dans les bâtiments un fumier qui n'est ensuite épandu que sur les champs et les prés de fauche. Dans ces conditions la fertilité des pâturages ne peut que décroître. 


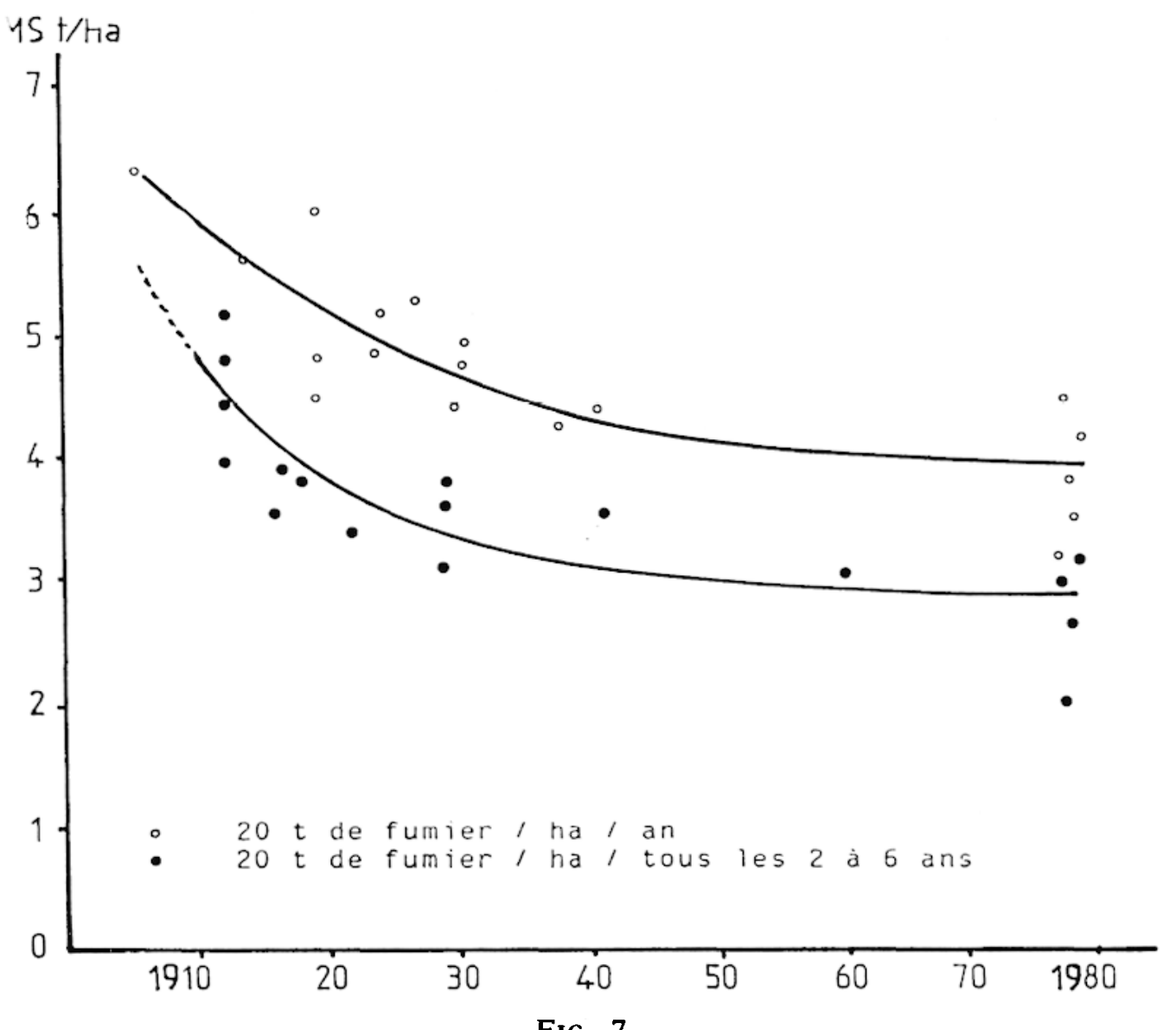

FIG. 7

Evolution de la productivité des prés de fauche selon la dose d'abandon de la culture (d'après F.-X. de Montard et al.. 1981).

L'analyse du couvert végétal (fig. 8) révèle et situe ces modalités d'évolution des surfaces pastorales après l'abandon de la culture. Au cours de cette évolution, la végétation des parcelles passe par un maximum de la valeur pastorale et leur fréquentation par les animaux est alors la plus intense (Balent, 1984; Balent et Duru, 1984). Tout se passe donc comme si les éleveurs tiraient au mieux partie de leur territoire pastoral. En fait, en l'absence de fertilisation, les très forts prélèvements des animaux dégradent rapidement la fertilité de ces parcelles; la valeur pastorale et l'appétence des pelouses baissent et, avec elles, la fréquentation par les animanx. En l'absence de gardiennage, les pressions de pâturage importantes se reportent alors sur de nouvelles parcelles dont la valeur pastorale t-st à son maximum.

Il existe donc un flux continu de parcelles évoluant d'un état initial (champ) vers un état final (pâturage pauvre). Au cours de cette évolution les éleveurs utilisent au maximum, mais sans la renouveler, l'importante fertilité de ces parcelles liée à leur ancien mode de mise en valeur (culture ou fauche). Cette rente heritée se manifeste donc à la fois dans la constitution des réserves hivernales, oì elle permet 
d'obtenir un surplus de récolte en regard des fumures apportées, et dans le pâturage, durant la période de vaine pâture, oì lcs surfaces présentant la meilleure valeur pastorale sont les plus utilisées par les troupeaux de la commune.

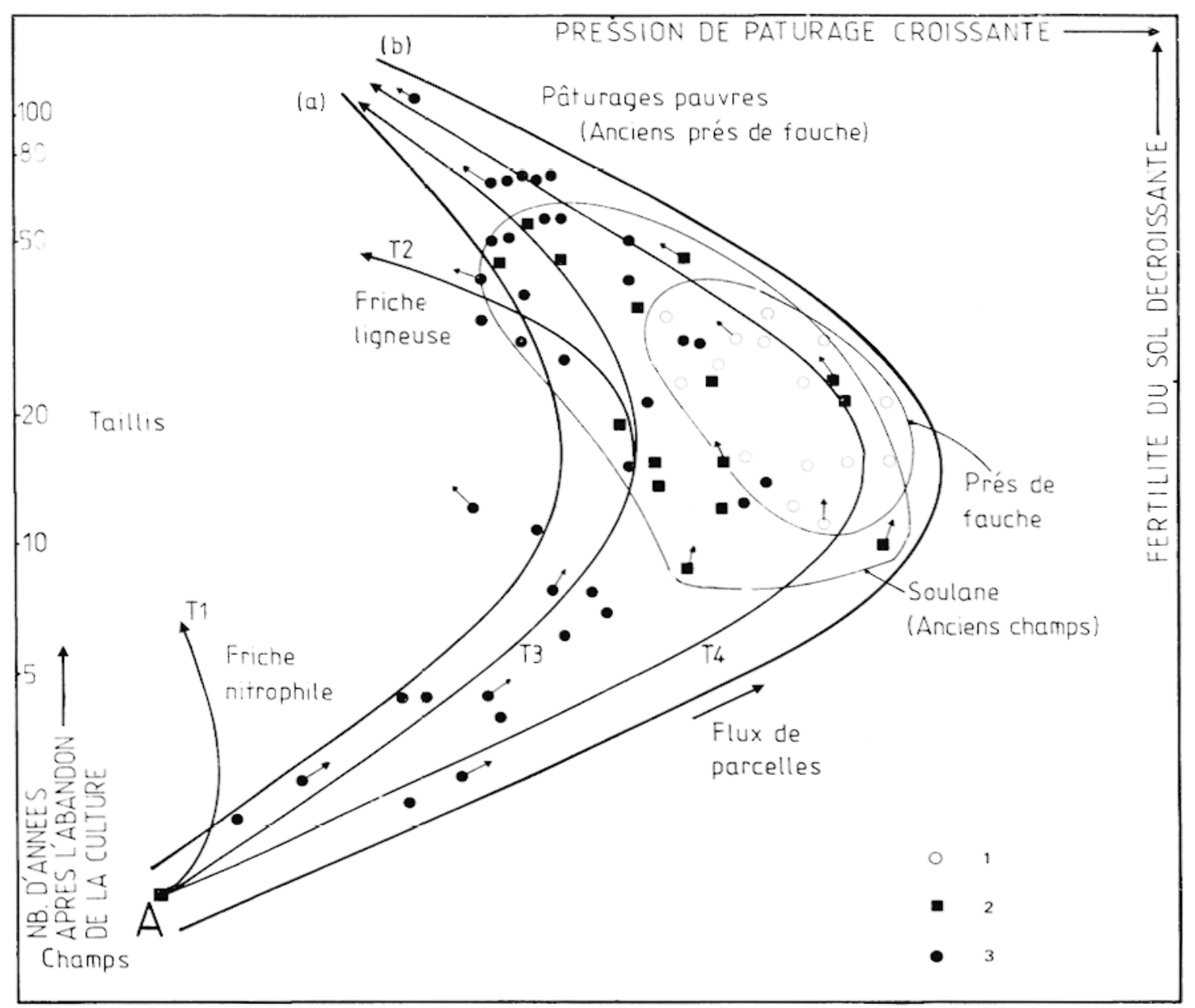

FIG. 8

Dynamique d'évolution du territoire pastoral :

relations entre fertilité du sol et fréquentation par les troupeaux. D'après Balent, 1984.

T1, T2, T3, T4 : Trajectoires d'évolution possibles pour une parcelle A après son abandon :

1. Stations sur prés de fauche. -2 . Stations en soulane. -3 . Autres stations.

\section{La désorganisation des pratiques de pâturage et ses conséquences}

1. Le contrôle "communautaire " des pratiques pastorales au $X I X$ " siècle.

Au cours du XIX" siècle, demeurent les traces locales, à Oô et dans la plupart des autres communes, d'une réglementation précise de 
l'usage du territoire pastoral (Dunor, 1979). Jusqu'en 1830, les réglements portent sur l'essentiel, notamment sur les dates de prohibi-

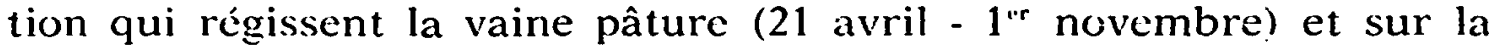
délimitation globale des grands quartiers de pâturages communaux; mais aussi, sur l'usage d'aller faucher sur la montagne (estive), ainsi que sur la délimitation du quartier de pâturage le plus riche réservé à l'engraissement des bovins destinés à la vente. La vie de la communauté, sa cohésion interne, reposent en fait sur une tradition orale cie droits et d'obligations, règles coutumières qui donnèrent lieu ì une expression écrite dans les cahiers de délibérations municipales - qui sont corrélatifs du droit coutumier -, quand elles furent menacées par le développement des différences sociales, la pression démographique, l'augmentation des parsans sans terre et les pressions exercées de l'extérieur de la communauté notamment par l'Etat (promulgation du Code forestier, politique de reboisements). Ainsi. à partir de 1880, la complexité des règles d'usage du territoire communal sera-t-elle précisée par écrit; elles touchent l'ensemble des activités agropastorales: règlements relatifs à l'activité pastorale (coupes fourragères, vaine pâture, cantonnement des animaux, activité des pâtres) et à toute autre pratique communautaire. Ces règles notifient. à partir d'un découpage du territoire en quartiers de páturage, les modalités de leur utilisation. Sont définies les catégories d'animaux admises selon les saisons et les périodes, la circulation des troupeaux sur les parcours, les modalités de gardiennage, ainsi que les droits et devoirs des familles. "Ces minutieux règlements sont promulgués et renouvelés régulièrement par chacune des communautés; fondés sur des pratiques sans doute multiséculaires et sur une connaissance remarquable du terrain, ils etablissent de subtils distinguo entre les diverses espèces animales sclon leur catégorie et leur fonction" (Soulet, 1981).

a. "Communauté " et propriété: une "communauté " peut se définir par un nombre déterminé de maisons exprimant un équilibre entre une population et un territoire (Assier-Andrieu, 1981). En offet, tout se passe comme si la communauté cherchait à établir un modèle garantissant l'équilibre entre ressources végétales nécessaires à l'alimentation humaine et ressources nécessaires pour nourrir le bétail indispensable à l'agriculture.

Si cette logique garantit la mise en valeur maximale du milieu, elle le fait sans remet1re en cause le partage des ressources, car elle est fondće sur la propriété privée de la terre et du bétail. La communauté constitue donc l'instance sociale de régulation de l'ensemble cu sytème agro-pastoral; mais cette régulation se fait au profit des familles possédantes. En effet, de grandes différences sociales existent cans cette collectivité montagnarde entre proprićtaires fonciers et paysans sans terre. Cependant la grande majorité des familles posséclantes dispose de biens à peu près équivalents. Lés "gros » proprićtaires sont des exceptions: à Oô, en 1838, un seul entre dans la 
catégorie des "hautes taxes", c'est-à-dire qu'il possède plus de 30 U.G.B. (6). Les règlements pastoraux traduisent une logique de gestion de l'esface visant à assurer la survie du plus grand nombre d'habitants et la reproduction des ressources en même temps que les privilèges des familles possédantes. Ils attestent de la domination des "petits bourgeois" (7) très largement majoritaires darıs cette commune; maîtres du Conseil municipal, ils prennent leurs décisions $\epsilon n$ invoquant sans cesse leur souci d'être équitables, leur volonté de garantir des droits "égaux et uniformes » à tous les usagers, la né. cessité de faire disparaître les "inégalités choquantes ». C'est ainsi qu'à. la fin du XIX" siècle, et en fonction des inquiétudes relatives aux pâturages de la commune "consommés sans ménagement et qui peuvent à peine fournir la nourriture des bestiaux ", les règles de gestion deviennent très strictes et le souci d'exclure les animaux "venus d'ailleurs " est la préoccupation dominante des délibérations. L'objectif est alors de limiter au maximum les bêtes extérieures introduites au pâturage par les paysans les plus pauvres, au moven de contrats de "gazaille» (8). Pour les gens sans terre (17 maisons sur 70 en 1886), ces contrats constituent pourtant le seul moyen d'obtenir l'argent nécessaire à l'achat des céréales qui leur font défaut. S'opposer à la gazaille, comme cela fut le cas dans d'autres communes, c'était prendre le risque de provoquer une révolte. En la taxant fortement, mais sans l'interdire, les propriétaires fonciers ont trouvé un moyen de limiter leurs propres charges financières et d'eviter le' risque de rebellion, tout en instaurant un mouvement d'exclusion "des pauvres et des marginaux".

b. Droits et devoirs des familles. Le nombre d'animaux que chaque "ayant droit " est autorisé à mettre sur les pâturages communaux est précisé dès 1827 . Il ne suffit plus pour cela de vivre au village ct d'être chef de famille: il faut être "propriétaire " (et pouvoir iustifier des titres de propriété devant un agent communal). Si durant la période de vaine pâture le nombre d'animaux pouvant en bénéficier, par propriétaire, reste indéterminé (l'enjeu économique étant la fumure et non la nourriture du bétail), le parcage des ovins dans les champs en mai et juin et l'usage des communaux sont très réglementés. Il fait. l'objet de précisions des droits par famille sclon les catégories d'animaux et le quartier concerné, en plus d'une limitation globale estimée à « 3 UGB par famille en plus des bêtes qui auront passé l'hiver dans: les étables » (9).

L'équilibre du système repose sur deux dispositions. Premièrement, le nombre de "bêtes à laine" mises au pacage est défini par mai-

(6) A la fin du XIX" siècle, une Unité Gros Bétail équivaut à 10 bêtes à laine (aujourd'hui le rapport est de 1 à 7). Cela atteste probablement de la petite taille et de la sous-alimentation des ovins, les parents pauvres du systèmc.

(7) Cahiers de délibération du Conseil municipal (1883).

(8) Droit qui autorise les habitants de la commune à introduire sur les parcours du bétail étranger.

(9) Cahiers de délibération du Conseil municipal. 
sen proportionnellement au nombre d'hectares possédés ou exploités (non compris les parcelles closes ou non sujettes à la vaine pâture). Les ovins "servant à l'engrais" des champs, cette mesure vise à assurer le maintien d'un nombre d'animaux suffisants pour reprocluire la fertilité des parcelles, condition nécessaire à la vie des familles, mais aussi à limiter la gazaille pratiquée par les familles pauvres. Les règles de participation à l'usage des biens communaux relèvent de la règle des "foins et pailles" qui conçoit le pàturage sur les communaux comme un "accessoire de la proprićtć privée et une sorte de prolongement des exploitations fourragères" (Parain, 1971).

Deuxièmement, les droits de dépaissance sur les pâturages servant à l'engraissement des animaux " clestinés au commerce "sont précisés par famille et par périodes autorisées pendant l'été. Cet arrêté garantit à tous les propriétaires une certaine égalité face aux activités commerciales; en cela il freine le developpenent de l'ćlevage, mais il permet au plus grand nombre d'accéder à l'échange marchand, leur permettant de satisfaire les besoins non couverts par les activités dominantes d'autosubsistance.

c. Quartiers de pâturage et gardiennage. La précision des droits de dépaissance traduit la recherche d'une adéquation entre iypes d'animaux et types de surfaces, entre charge animale ei type de pâturage. Il s'agit, selon les propres terınes du Conscil : "d'assurer une dépaissance régulière et méthodique du pâturage" ". Nous avons identifić 22 quartiers pour 13 catégories d'animaux constituátnt des "troupes" communales (10). Il est en effet précisé qu" "afin d'assurer lá régularité des services communaux, toute bête devra pacager avec celles de son âge et espèce " et sera soumise à un gardiennage. Aucune catégorie d'animal (chèvres comprises), n'est autorisée à pacager librement sur les terres communales. Le gardicinnage peut être collectif ou indivicluel (il y eut jusqu'à 7 bergers à Oô). Au vacher et au berger communaux embauchés tout au long de l'année (et soumis à l'autorité du Conseil), s'ajoutent pâtres et bergers chargés de la surveillance' estivale, ainsi que ceux qui surveillent les troupes "particulières". Certains parcours sont réservés aux «bergers particuliers», d'autres aux troupes communes, mais la priorité reste donnée à la préservation cles intérêts collectifs, ce qui laisse peu de place aux initiatives privées, même hors des terres communales. Il fut précisé, en 1913, que conformément aux usages "tant anciens que modernes", il est défendu à tout propriétaire individuel de former un troupeau qui puis-

(10) Il s'agit des troupeaux de «bétail à laine», de bovins destinés au commerce (des vaches laitières), du jeune bétail à cornes (de moins de 3 ans), des veaux de lait, des bêtes à cornes (gros bovins), de brebis d'engrais, des chevaux de louage, des pouliches de 3 ans, des bêtes de travail (paires de vaches servant au labour, bêtes employées au transport de bois de chauffage, chevaux scrvant au fumage des champs), des chèvres, parfois des porcs, enfin du troupeaux des bêtes malades (reconnues malades par l'autorité locale). Sources: cahiers de délibération du Conseil municipal. 
se porter préjudice aux intérêts des troupeaux communaux. Tout propriétaire reste libre de ne pas faire partie de celui-ci, mais il doit alors emprunter des parcours réservés «aux particuliers", ou bien ne faire pacager son troupeau (quelle que soit la catégorie l'animaux) qu'après le passage du troupeau communal.

d. Une structure sociale régie par les stratégies de préscrvation jamiliale. Par tous les moyens, la "communauté "veille sur la stabilité foncière et le nombre de "maisons » et reproduit, par là même, les différences sociales. Les us et coutumes complètent ces dispusitions, au niveau de la famille, par des règles de transmission du patrimoine (Barrué-Pastor et al., 1982) qui garantissent le caractère inaliénable cle la propriété familiale. Ces règles sont particulièrcment rigides dans la commune d'Oô. Un dispositif complexe a permis d'éviter les partages jusqu'à une période très récente. L'héritage va à l'aîné des garçons (alors que dans la plupart des vallées pyrénéennes il va à l'aîné, garçon ou fille); les successions ne se règlent qu'à la mori cles parents et aucun partage d'utilisation n'est possible. A cela vient s'ajouter un phénomène de régulation des naissances: aucune des familles possédantes n'a plus de 2 enfants alors que les autres en ont jusqu'à 9. Les traces de l'endogamie villageoise, alors favorisée par les familles, sont encore visibles aujourd'hui à travers les multiples liens de parenté qui lient les exploitants du village. Un tel système assure la reproduction des ressources, mais aussi des rapports sociaux dans la communauté, qui sont caractérisés par une relative stabilisation des pratiques et des biens détenus par "maisons ». Cette loi de préservation des patrimoines multiplie par ailleurs, à chaque génération, la catégorie des paysans sans terres, puis les élimine. La grande majorité des familles qui ont réussi à se maintenir dans l'agriculture jusqu'à maintenant sont issues des lignées des notables, conseillers municipaux dans la dernière moitié du XIX" siècle, et dont on retrouve les signatures dans les cahiers de délibération.

\section{Les pratiques actuelles de pâturage.}

On distingue deux phases dans le calendrier de pàturage des troupeaux en vallée d'Oô, comme dans tout le Haut Comminges. La première est dominée par le caractère collectif des troupeaux et des pratiques de gardiennage. En estive, il n'y a que deux troupeaux. un pour les ovins (1 800 tètes environ) et un pour les bovins ( 300 têtes), gardés par un berger et un vacher engagés par le "groupement pastoral »(11). Pendant les demi-saisons, à la fin du printemps et en automne, l'ensemble des troupeaux de la commune (une dizaine pour

(11) Les éleveurs de bovins et ovins de la commune ont décidé lors d'unc assemblée générale, le 2 avril 1976 de former un "groupement pastoral ". Ils ont ainsi sollicité un bail de location des terrains communaux fréquentés par leurs troupeaux pendant l'été (estives); bail de 3 ans jusqu'en 1979, puis renouvelable chaque année. La création du G.P. est essentiellement un moyen d'obtenir. des subventions pour effectuer les aménagements qui devenaient urgents (entretien des chemins pastoraux, construction de cabanes des pâtres). 
les bovins et une demi-douzaine pour les ovins) sont regroupés en deux troupeaux, (un par espèce), gardés à tour de rôle par les ćlceveurs ou bien par le berger et le vacher d'estive. Une deuxième phase est celle de vaine pâture entre le $1^{\text {"r }}$ novembre et la fin avril. Elle est actuellement marquée par la diversification des pratiques de pâturage et l'émergence de stratégies individuelles de gardiennage. La principale différence avec le XIX" sic̀cle réside en effet dans la disparition de bergers et vachers communaux présents toute l'année sur la commune. Cela a conduit à une clégradation progressive de l'efficacité du gardiennage pendant la vaine pâture hivernale. Or, c'est la période qui traduit la plus forte pression de la collectivité sur l'individu dans la mesure où la relation entre la propriété et l'usage inclividuel du sol est temporairement abolie. Ce décalage entre usage individuel et communautaire de l'espace pastoral est une des expressions de la mutation des systèmes de production pyrénéens.

a. Circulation des troupeaux dans la valléc.

Circuits de pâturage: les modalités de circulation des troupeaux sur les pâturages de fond de vallée soulignent cette opposition entre le collectif et l'individuel. Pendant la périocle hors estive, les troupeaux accomplissent le plus souvent des circuits journaliers à partir des bâtiments d'élevage. En demi-saison il peut s'agir de granges "foraines" (c'est-à-dire éloignées du village), en hiver des étables et bergeries attenantes aux habitations du village. L.es observations et les enquêtes auprès des agriculteurs, chaque semaine, pendant trois années consécutives, permettent d'identifier une clouzaine d'itinéraires-types de circulation des troupeaux individuels ou collectifs sur le territoire pastoral. Ces circuits-types sont communs aux espèces ovines et bovines. Selon les cas, le parcours du circuit-type est guidé par un gardien, ou par l'éleveur, qui conduit le troupeau sur les pâturages le matin et lui "donne le tour". c'est-à-dire l'oriente sur le circuit qui sera suivi toute la journée par les animaux. Une des conséquences de cette pratique est que le pâturage peut être mixte. Au cours d'une journée, une même portion du territoire pastoral peut, en effet, être pâturée simultanément par des troupeaux des deux espèces (ovins et bovins) "lancés" sur le même circuit par leurs propriétaires. C'est une des originalités des systèmes pastoraux des hautes vallées pyrénéennes.

Les circuits-types identifiés (fig. 9) utilisent l'ensemble des ressources pastorales de fond de vallée: les champs (2), les anciens champs $(6,7)$, les prés de fauche $(3,4)$, les anciens prés de fauche $(8)$, les communaux, en association avec des anciens prés de fauche (9-10) ou seuls (11-12).

Les circuits 1 et 2 associent champs et prés de fauche. Le circuit 2 joue un rôle particulier en automne, après la récolte des maïs : les bovins y sont conduits pour pâturer les cannes sur pied. Les parcelles oì. la culture a été récemment abandonnée bénéficient du passage dcs animaux, car le piétinement élimine les espèces de la friche et favorise l'implantation des espèces fourragères (Balent et Duru, 1984). 
Les circuits sur prés de fauche (3-4-5) intéressent aussi, le plus souvent, une partie des anciens prés. La double utilisation des parcelles (fauche, pâture) et les soins dont les prés de fauche sont l'objet (fumure) font de ces circuits, comme nous le verrons plus loin, les zones les plus fréquentées par les animaux en hiver et surtout au début du printemps.

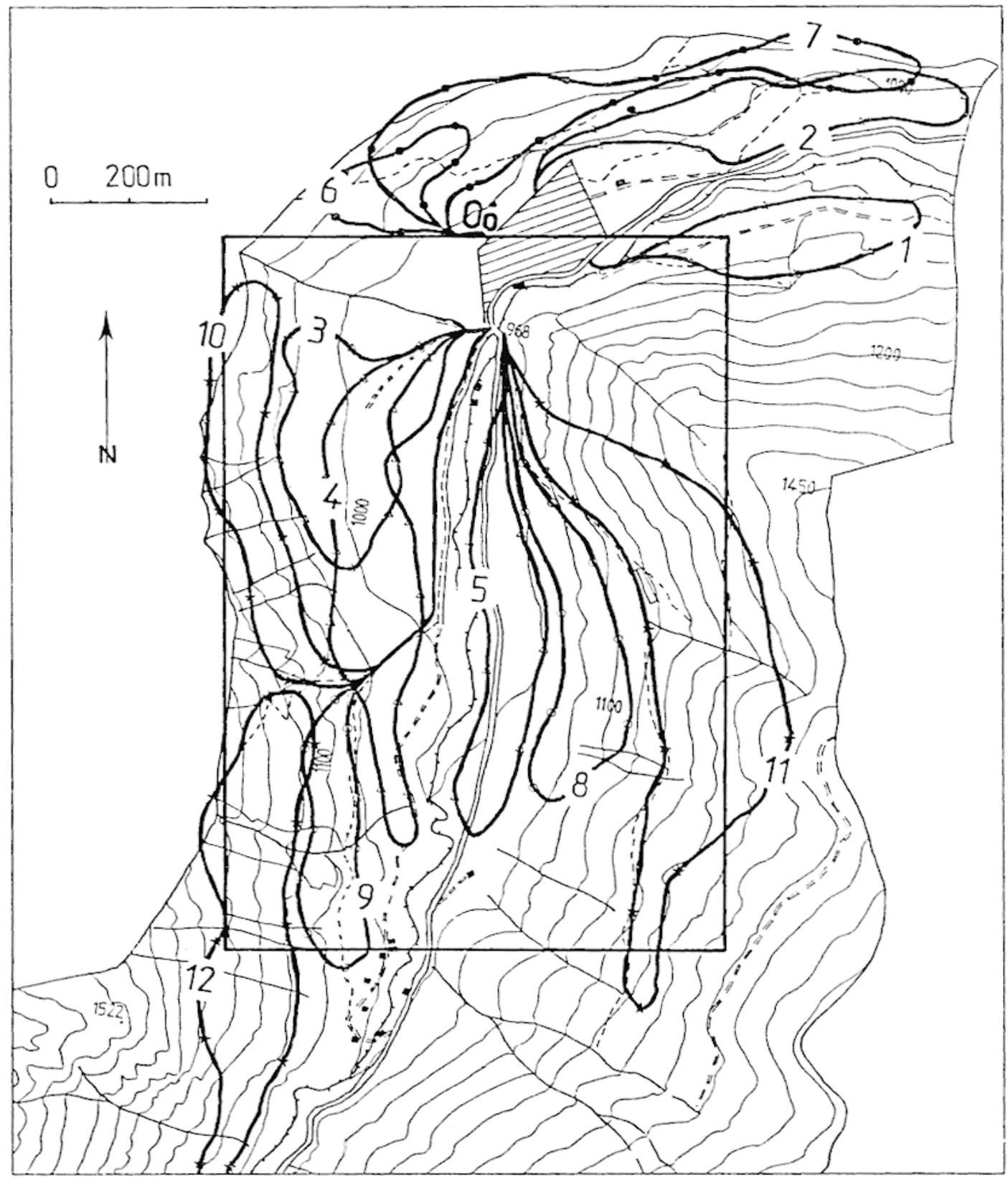

FIG. 9

Les circuits de pâturage hors estivage dans la vallée d'Oô. 1 (Sur fonds oro-hydrographique, chaque circuit étant numéroté séparément de 
Les circuits sur les anciens champs (6-7) sont très importants aussi. Ils sont utilisés tout au long de la période hors estive, mais ils jouent surtout un rôle capital au cœur de l'hiver. Les terroirs d'anciens champs sont en effet situés en "soulane" (versant exposé au sud). Après de fortes chutes de neige, l'ensoleillement lavorise leur déneigement précoce et ce sont alors les seules surfaces pastorales utilisables.

Deux des circuits sur les communaux (9 et 10) débordent sur une partie des anciens prés de fauche. La proximité des prés de fauche et l'absence de clôture interdisent leur utilisation sans gardiennage en automne à la période où la réglementation protège les régains. Les deux autres (11 et 12), ne sont fréquentés qu'en circuits de ciemisaison (pré et post-estive). Ils sont surtout utilisés par des troupeaux assez importants sous la surveillance d'un berger ou d'un vacher (la distance au village est grande, le relief tourmenté et la visibilité mauvaise). Leur flore est très dégradée (nombreux ligneux) mais cvolue très lentement car leur productivité est faible.

Utilisation des circuits de pâturage (fig. 10). Sur trois années d'observation, on constate que la règle de la vaine pâture est bien respectée et que les dates d'application varient peu, pouvant être légèrement avancées à l'automne lors d'une chute de neige précoce en estive (1979) ou retardées au printemps à la suite d'un hiver particulièrement rigoureux (1981). De grandes lignes d'utilisation du territoire pastoral se dégagent. Les prés de fauche sont tiès pâturés au printemps. La pousse de l'herbe y est plus précoce que sur les autres surfaces pastorales, en raison de leur fertilité élevéc et permet aux animaux de reconstituer leurs réserves corporelles à la sortie de l'hiver (Gibon, 1981). L'hiver, les circuits de soulane sont les plus utilisés, pour les raisons déjà évoquées; en 1981, année d'enneigement important, ils furent la seule possibilité de pâturage pendant deux mois et demi. Cependant ces zones pentues, sourent aménagées en terrasses sont très fragiles et leur fréquentation importante, par les bovins en particulier, pose de nombreux problèmes pour leur avenir. Le regroupement des animaux en gros troupeaux (700-800 ovins et 200 bovins) ne se produit qu'au printemps et en automne, avant et après l'estive; à ces périodes les animaux utilisent exclusivement les zones intermédiaires et les communaux. Au printemps ils profitent d'un pâturage de qualité en raison d'une pousse de l'herbe plus tardive et en automne de reports sur pied abondants. En automne, le nombre d'animaux diminue progressivement car une partie est descendue en plaine. En hiver, le nombre des bovins au pâturage est très faible. Par contre, l'ensemble des ovins pâture dès que les conditions métćorologiques le permettent.

Sur chacune des trois années, l'ensemble des circuits est utilisé et des règles générales se dégagent : l'utilisation des quartiers de prés de fauche au printemps avant la prohibition, (utilisation de la pousse précoce des prés pour la remise en état des animaux), de la soulane: en hiver (adaptation aux aléas climatiques hivernaux) et des commu- 

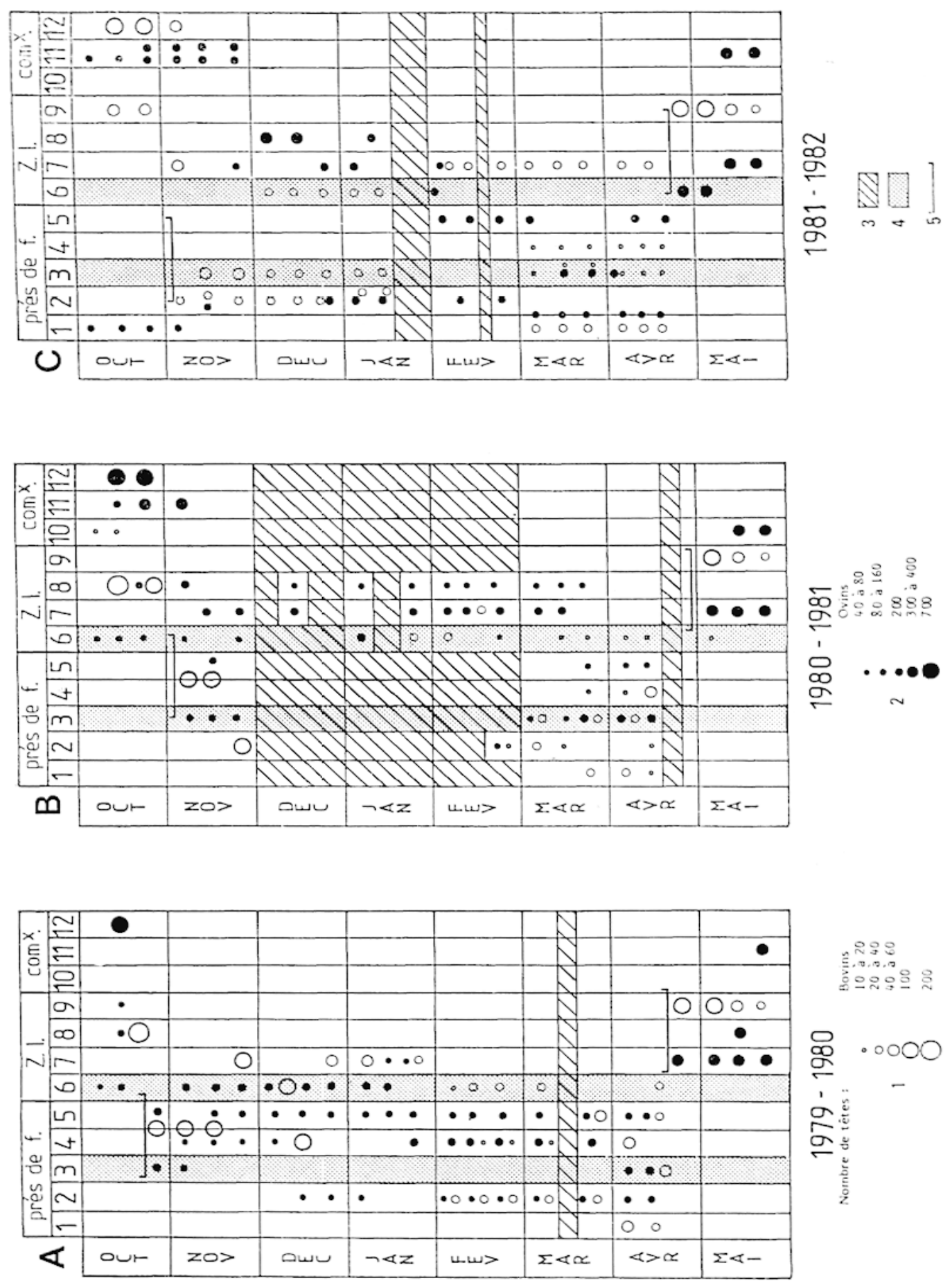

Fig. 10

Modalités d'utilisation des circuits de pâturage.

1. Bovins. - 2. Ovins. - 3. Neige au sol. - 4. Circuits dont l'usage est précisé au tableau 3 (infra). - 5. Dates de début et de fin de la vaine pâture. 
naux avant et après l'estive (adaptation à des contraintes agronomiques liées à l'altitude). Les variations climatiques interannuelles modulent cette utilisation. Cependant la taille et la composition des troupeaux qui utilisent chaque circuit de pâturage varient d'une année sur l'autre. Les particularités climatiques ne suffisent pas à expliquer ces variations. Il faut invoquer des différences dans la conduite au pâturage des troupeaux de chaque éleveur. Un même circuit n'est pas utilisé à la même époque d'une année sur l'autre; ce ne sont pas toujours les animaux d'un même éleveur qui exploitent un circuit donné à une époque donnée, ni le même type d'animal (lé páturagé peut être mixte). Ainsi le circuit 3 est-il situé sur les prés de fauche qui sont les pâturages les plus utilisés et le circuit 6 dans la soulane qui est une zone stratégique pendant l'hiver (fig. 10 et tabl. 3).

TABL.EAL 3

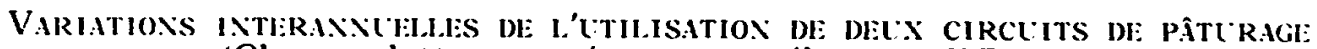
(Chaque lettre représente un éleveur différent, $\mathrm{C}$ représente un troupeau communal).

\begin{tabular}{|c|c|c|c|c|c|c|c|c|c|c|c|c|c|}
\hline & & \multicolumn{6}{|c|}{ CIRCUIT 6} & \multicolumn{6}{|c|}{ CIRCUIT 3} \\
\hline & & \multicolumn{2}{|c|}{1980} & \multicolumn{2}{|c|}{1981} & \multicolumn{2}{|c|}{1982} & \multicolumn{2}{|c|}{1980} & \multicolumn{2}{|c|}{1981} & \multicolumn{2}{|c|}{1982} \\
\hline & & & & & B & & & 0 & & 0 & & & B \\
\hline \multirow{2}{*}{ AUTOMNE } & Début & $\mathrm{A}$ & - & B & - & - & - & $\mathrm{D}$ & - & - & - & - & $\mathrm{x}$ \\
\hline & Fin & A & C & B & - & & - & - & - & $\mathrm{C}$ & - & - & $\mathrm{x}$ \\
\hline \multirow{2}{*}{ HIVER } & Début & $A$ & - & A & $\mathrm{xz}$ & - & C & - & - & - & - & - & $\mathrm{x}$ \\
\hline & Fin & - & $\mathrm{x}$ & - & - & A & - & - & - & $\mathrm{EC}$ & $x$ & - & - \\
\hline \multirow{2}{*}{ PRINTEMPS } & Début & - & & - & $Y$ & - & - & E & $x$ & $\mathrm{EC}$ & $\mathrm{x}$ & EC & $Y$ \\
\hline & Fin & - & - & - & Y & $\mathrm{AC}$ & - & - & - & - & - & - & - \\
\hline
\end{tabular}

b. Stratégies individuelles et pratiques de gardiennage. L'évolution déterminante des pratiques de gardiennage tient à la fin de l'obligation de gardiennage et de la présence d'un berger et d'un vacher communaux toute l'année. Jusque dans les années 1980, les éleveurs ont, pendant la vaine pâture, regroupé les animaux en un troupeau communal par espèce, gardé à tour de rôle en fonction du nombre d'animaux mis dans le troupeau. Mais des intérêts contradictoires (par exemple, tirer au maximum sur lc pâturage ou profitcr de stocks de foin important) ont entraîné l'abandon de cette prati- 
que. Chaque éleveur développe sa propre stratégie selon ses objectifs, qu'il tienne à conserver l'individualité de son troupeau ou qu'il recherche des associations avec d'autres, qu'il assure lui-même la surveillance de son troupeau, qu'il le confie à un berger ou un vacher, ou qu'il abandonne le gardiennage.

Les problèmes se posent différemment à deux périodes distinctes. Pendant la période de prohibition des prés de fauche entre la fin d'avril et le début de novembre, les pratiques communautaires dominent encore largement. Les éleveurs profitent de la présence du berger et du vacher d'estive pour faire garder leurs animaux en les intégrant dans les troupes communales ou cantonnent les animaux dans des zones clôturées.

Pendant la vaine pâture la situation est plus complexe et aboutit à l'abandon presque total du gardiennage. La diversité sociale des propriétaires en semble une des causes principales. Pendant cette période, où la liberté d'utilisation de l'ensemble du territoire de la commune par les troupeaux est totale, on assiste à de nombreuses tentatives d'appropriation individuelle de circuits de pâturage.

La stratégie d'une minorité d'éleveurs consiste à s'approprier systématiquement un circuit de pâturage, en se faisant reconnaître tacitement la jouissance d'une zone qui ne présente pas d'intérêt particulier pour les autres éleveurs car difficile à garder et à ressources limitées. C'est ainsi, par exemple, que pendant les trois annces d'observation des circuits de pâturage le même éleveur d'ovins, un double actif possédant une vingtaine de brebis, "colonise " de nıanière permanente d'octobre à juin un terroir d'anciens champs sur terrasses très pentues et quasiment reconquis par les boisements.

Pour d'autres, en général propriétaires cles troupeaux les plus importants ( 200 ovins ou 20 bovins), les tentatives d'appropriation enregistrées correspondent à une volonté d'utiliser leurs propres parcelles pour bénéficier à la fois des fruits de leur travail et des apports de leurs animaux, en ce qui concerne la fumure et l'entretien du pâturage. Il s'agit parfois d'augmenter la maîtrise individuelle de la reproduction et de l'état sanitaire du troupeau ou bien de limiter la surveillance des animaux; dans certains cas encore de limiter les relations d'interdépendance avec les autres éleveurs de la commune. Même si les circuits qu'ils utilisent, varient d'une année et d'une saison sur l'autre, leurs troupeaux ne se mélangent pas (hormis quelques "accidents " par absence de gardiennage). Certains pratiquent l'hivernage partiel en plaine, leurs ressources en fourrages et leurs capacités d'accueil étant insuffisantes, et embauchent pour cela un berger ou un vacher qu'ils conservent en dehors de la période d'hivernage, afin d'augmenter leur degré d'autonomie par rapport aux autres éleveurs. Pour la plupart de ceux qui font pâturer leur troupeau individuellement, tout se passe comme si le premier arrivé sur un circuit en début de saison avait une priorité de fait sur son usage jusqu'à ce que les conditions générales de pâturage changent (vaine 
pâture, neige, etc.), auquel cas, une nouvelle répartition se met momentanément en place.

Les propriétaires de petits troupeaux pratiquent plus volontiers une stratégie de regroupement; mais on observe une grande diversité des alliances entre éleveurs. Les causes en sont dans la diversification des types d'éleveurs et dans la crise de la société montagnarde qui aggrave les conflits. Elles sont de trois types. Un premier se réfère au projet professionnel de l'exploitant : ceux qui sont en phase d'expansion, ou qui développent leurs efforts dans le sens de la maîtrise de leur système d'élevage et de l'amélioration des conditions sanitaires, ne tiennent pas à voir leurs bêtes mêlées à celles d'exploitants en phase régressive et moins exigeants sur la productivité et l'état sanitaire de leurs animaux. Un second se réfère aux disponibilités en temps de travail: comme dans la première catégorie, c'est une certaine communauté d'intérêts qui a poussé quatre des six doubleactifs du village à constituer un troupeau commun. Un dernier se réfère à la qualité des relations que les exploitants entretiennent entre eux : au-delà des conflits, une certaine permanence dans les relations familiales se maintient; ainsi rencontre-t-on des troupeaux collectifs regroupant des animaux appartenant à des cousins au premier degré.

Le caractère dominant de ces alliances reste leur grande instabilité inter-annuelle et aussi intra-saisonnière. Aucun des critères retenus pour les préciser n'engendre de déterminisme véritable et durable quant aux rapports sociaux. La grande variabilité des pratiques et des choix individuels se rapproche davantage d'un ajustement d'année en année, parfois au jour le jour, donc d'un pilotage à vue qui évoquerait la tactique plutôt que la stratégie. Chacun pare au plus pressé en choisissant le "moindre mal ", selon la conjoncture. Ainsi ces alliances, ne traduisent-elles pas une volonté de regroupement guidée par une certaine cohésion sociale, mais plutôt une réaction mouvante face à l'adversité qui engendre une stratégie d'ajustement définie par la négative.

\section{c. Conséquences de l'évolution des pratiques pastorales.}

Les conséquences agronomiques et écologiques de ces nouvelles pratiques constituent un excellent révélateur du niveau de cohérence actuel du système pastoral. Le choix individuel des circuits et l'abandon du gardiennage ont comme conséquence une mauvaise répartition des charges sur les pâturages, qui peut s'apprécier à différents pas de temps. A l'échelle de la journée la liberté laissée aux animaux du fait de l'abandon du gardiennage a une conséquence immédiate sur leur comportement spatial et alimentaire: les animaux sont attirés vers les parties du territoire pâturé ayant la meilleure flore et le maximum d'herve verte (Arnold et Dudzinski, 1978, Després et Soulas, 1981), c'est-à-dire, les prés de fauche et les anciens champs. A. l'échelle de l'année, la répétition de ce type de comportement fait que les animaux vont délaisser les zones marginales abandonnées 
depuis longtemps par la culture et la fauche et par la même accélérer le processus d'enfrichement et de fermeture de ces zones et concentrer leur fréquentation sur les surfaces les plus fertiles et les mieux entretenues. Ce que montre bien l'analyse des prélèvements effectués par les ovins et les bovins entre octobre 1980 et juillet 1981 (Balent et Charpenteau, 1984), qui signale la concentration territoriale des activités de pâturage (fig. 11). L'essentiel du prélèvement se situe sur les actuels prés de fauche, qui font l'objet d'une exploitation très intensive ( 1 à 2 fauches, plus 2 pâturages, l'un d'automne-hiver, l'autre de printemps), alors que le reste du territoire est peu parcouru au cours de l'année. Les parties les plus délaissées sont les communaux puis la zone d'anciens prés. A court terme (la journée), la concentration des animaux sur les meilleurs pâturages a une action bénéfique sur leur alimentation; mais à long terme (plusieurs années), la surexploitation des prés de fauche conduit à une baisse importante de leur fertilité et à l'enfrichement du reste du territoire communal.

Les conséquences sociales. Le développement de l'individualisme dans les pratiques des éleveurs aboutit à une conséquence grave: la pose de clôture sur un territoire où l'interdit la coutume de la vaine pâture. Au cours des ces dernières années, en effet, 14 parcelles, soit environ 6 hectares, ont été clôturées et font l'objet d'un usage privatisé de la part de six exploitants. Ces enclosures correspondent en général à deux types de situations: ou bien il s'agit de petits éleveurs pluri-actifs qui parquent des animaux pour la journée dans des parcelles dont la taille est suffisante pour assurer leur nourriture (c'est alors une façon de limiter le gardiennage) ; ou bien il s'agit de proprićtaires de gros troupeaux qui parquent les brebis mères suitées de très jeunes agneaux pour favoriser leurs contacts et les protéger de tout accident éventuel (l'objectif majeur est alors de faciliter les modes de fonctionnement et d'alléger la charge de travai?).

Ces appropriations, comme les fluctuations des alliances déjà évoquées, constituent des facteurs destabilisants. Leur émergence est reliée à la destructuration des règles d'organisation antérieures et à la diversification des «profils " d'éleveurs. Elles correspondent à une démarche strictement individuelle susceptible de remettre en cause les dernières pratiques communautaires. Si nous parlons de destructuration c'est qu'en effet les pratiques actuelles s'enracinent dans les pratiques antérieures. Nous en trouvons les traces dans le règlement de la vaine pâture et le découpage ciu territoire en circuits de pâturage en dehors des périodes d'estivage. Et ces anciennes pratiques n'ont fait l'objet d'aucune actualisation raisonnée par la collectivité. En l'absence d'outil de contrôle actif, elles ont perdu progressivement leur sens et leur impact. Par rapport au système antérieur, les centres de décisions se sont déplacés de la communauté vers les individus. Actuellement, la gestion de l'espace pastoral correspond à l'addition de stratégies d'éleveurs ayant des projets et des contraintes très diversifiés pour ce qui touche leurs exploitations. 


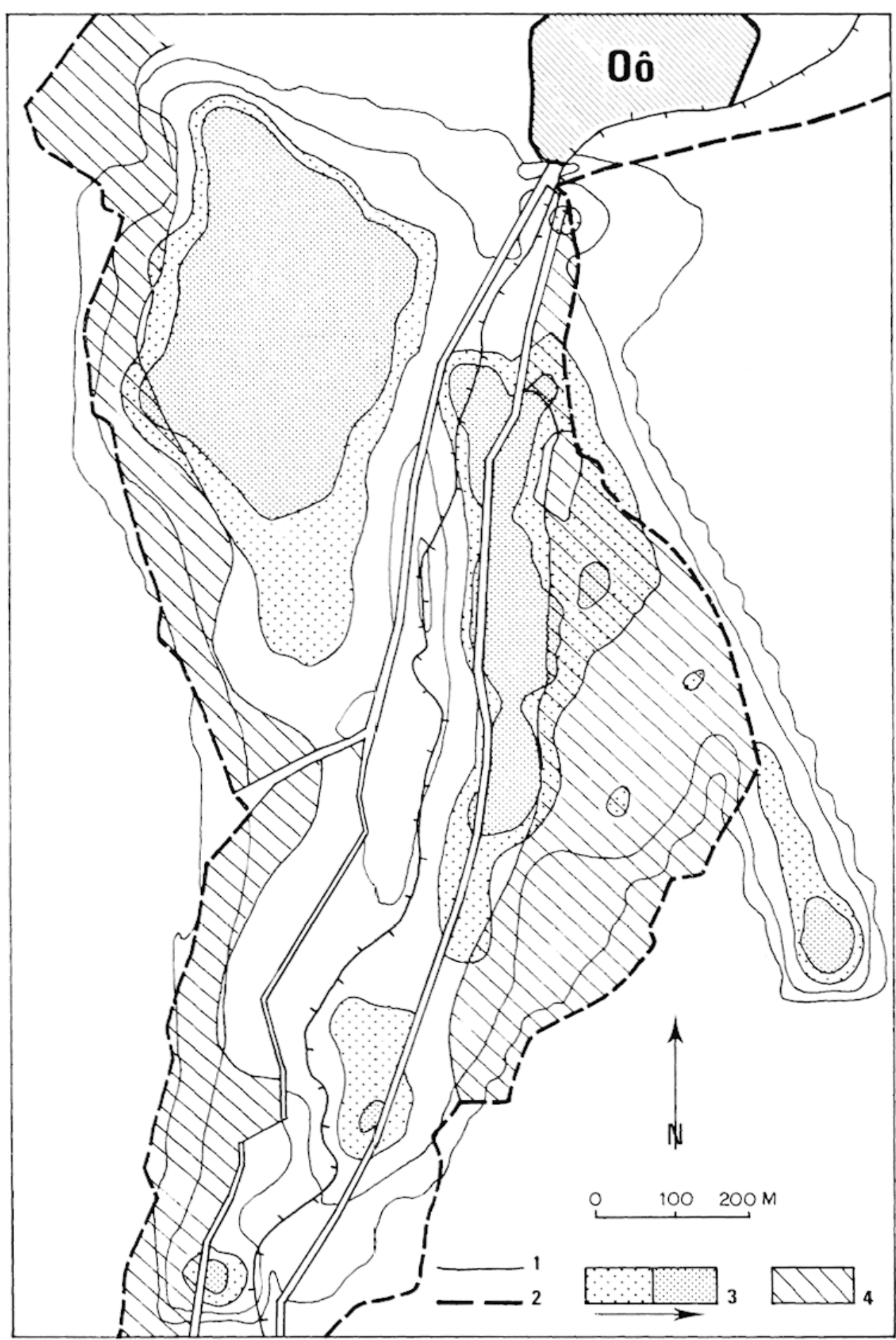

FIG. 11

Pression de pâturage sur les prés de fauche (hiver et début de printemps). (Pression de pâturage croissante selon l'intensité du grisé)

1. Courbe d'iso-intensité de pâturage. - 2. Limite du communal. - 3. Pression de pâturage. - 4. Zone intermédiaire. 
D'où la difficulté à construire un système de contrôle territorial qui leur assure, à long terme, la conservation de la maîtrise de cet espace et de ses ressources.

Cette situation traduit une destructuration des principes de l'organisation sociale qui articulent à la fois les rapports que chacun entretien avec le territoire et les rapports entretenus à l'intérieur d'un même groupe ou entre les différents groupes sociaux. Ici, les contradictions de la production agricole (techniques, économiques et sociales) se situent tant au niveau de l'exploitation (individu-familleexploitation) qu'au niveau de l'ensemble des différents groupes d'éleveurs intéressés à la maîtrise de l'espace. Dans ce contexte, les pratiques des uns et des autres entrent en contradiction, d'autant plus vivement, qu'elles s'inscrivent dans des impératifs dont les pas de temps sont très différents. L'individualisation des pratiques et l'abandon du gardiennage s'inscrivent dans la logique d'un court terme déconnecté de toute perspective de succession, et donc d'entretien du "potentiel " détenu. Ceux qui prônent un mode de gestion orienté vers un contrôle du territoire qui assurerait la conservation des ressources s'inscrivent dans la logique productive nécessaire à la viabilité de projets professionnels, notamment pour ceux qui sont en phase d'installation. S'il n'y a plus d'organisation d'ensemble de la gestion des pâturages, c'est qu'il n'existe plus de collectif suffisamment fort pour imposer une règle de fonctionnement. Nous avons là un des révélateurs les plus importants de la destructuration de la cohésion agricole de la collectivité locale montagnarde qui a de plus en plus de mal à jouer son rôle d'instance sociale de régulation des activités pastora!es. Cette caractéristique se généralise à l'ensemble des zones de montagne françaises (Pernet et Lenclud, 1977). Les causes en résident dans les principaux éléments qui la structurent et qui relèvent de deux pôles: les règles qui régissent le groupe domestique et celles qui régissent la collectivité nationale. C'est dans l'interférence de ces deux éléments qu'est le moteur du changement. Car la gestion des ressources pastorales et la reproductibilité des systèmes de production sont liées aux possibilités qu'ont les agriculteurs à assurer leur propre reproduction dans la collectivité montagnarde.

\section{Structures sociales et blocage foncier}

Le caractère du mode de mise en valeur d'un territoire découle de la capacité d'une structure sociale à en gérer les conditions d'accès et à en assurer le contrôle. Si l'entretien et la gestion des terres agricoles - qu'il s'agisse des zones à utilisation privée ou collective dépend de leurs conditions d'utilisation, c'est dans l'étude du statut juridique d'accès au foncier (droits d'usage) et dans le régime de la propriété privée qu'il faut en chercher les causes (Barrué-Pastcr et al, 1982). 


\section{Une modification des délimitations entre propriété privée et pâturages collectifs.}

La diminution de l'activité agricole s'accompagne, nous l'avons vu, d'un "glissement vers le bas " des surfaces utilisées qui entraîne de nouvelles délimitations territoriales des pratiques pastorales, notamment, une utilisation de parcelles privées en parcours collectif. Ces parcelles, mises à disposition du groupe local, ne peuvent être assimilées juridiquement aux terres communales, alors qu'elles ont été intégrées aux surfaces pastorales d'usage collectif. Elles représentent $55 \%$ de la surface cadastrée en propriété privée. Elles ne sont plus utilisées que sous forme de parcours collectifs et ne font l'objet d'aucun entretien de la part de leurs proprićtaires (fauche, drainage, entretien des terrasses, etc.). Ces parcelles sont passćes sans transition d'un mode d'utilisation intensif (fauche ou culture) à une utilisation du type de la "cueillette" des ressources fourragères par le pacage des animaux; leur évolution floristique est très rapide et va toujours dans le sens de l'appauvrissement de la valeur pastorale du couvert végétal et de l'embroussaillement. Or ces surfaces jouent un rôle stratégique dans l'alimentation des troupeaux all cour de l'hiver et en demi-saison: le problème de leur remise en valeur, ou tout au moins l'arrêt de leur évolution régressive, se pose avec urgence. Leur statut juridique (propriété privée) complique les possibilités d'intervention, car il faut, non seulement l'accord de tous les agriculteurs sur un projet d'aménagement, mais encore, et surtout. celui de tous les propriétaires (dont la plupart ne sont plus agriculteurs ou ont même quitté la vallée).

\section{Diversification de la typologie des propriétaires fonciers.}

Actuellement $46 \%$ des surfaces cadastrées appartiennent à des propriétaires qui n'ont aucune activité agricole. Parmi eux, si $35 \%$ étaient déjà propriétaires en 1954, 65\% d'entre eux ont acquis leur propriété depuis cette date (35\% par héritage, $30 \%$ par achat). $54 \%$ des superficies appartiennent à des familles qui ont conservé une activité agricole, mais le tiers est entre les mains d'exploitants retraités sans successeurs (tabl. 4). En fait les agriculteurs actifs ne maîtrisent que $40 \%$ des superficies cadastrées. (Barrué-Pastor, 1980, 1982). A ce titre, Oô se comporte comme la plupart des communes des zones de montagnes françaises, avec toutefois une résistance agricole très marquée, à l'origine des préoccupations relatives au processus de régression.

a. L'hétérogénéité nouvelle des exploitants. La déprise agricole se traduit par une diversification des propriétaires fonciers mais aussi des exploitants. Sur les 16 exploitants que compte aujourd'hui la commune, plus de la moitié ont au moins 60 ans (la moyerne d'âge est de 50 ans), et la quasi totalité de ceux qui ont entre 35 et 50 ans 
TABLEAC 4

TYPOLOGIE DES PROPRIÉTAIRES FONCIERS FNTRE 1954 FT 1980 (En 1980, $14 \%$ des retraités ont conservé une activité agricole)

\begin{tabular}{|l|c|c|c|}
\hline $\begin{array}{l}\text { en surfe } \\
\text { Surface }\end{array}$ & Actifs agricoles & Retraités agricoles & $\begin{array}{c}\text { Propriétaires non } \\
\text { agricoles }\end{array}$ \\
\hline 1954 & 62 & 29 & 9 \\
\hline 1980 & 40 & 37 & 23 \\
\hline Variation & -35 & -28 & +155 \\
\hline
\end{tabular}

sont pluri-actifs. Deux jeunes agriculteurs sont en phase d'installation. Les structures foncières sont d'une grande stabilité $(85 \%$ des exploitations n'ont enregistré aucune évolution des superficies en propriété depuis 1954) et traduisent encore la réalité du XIX" siècle. L'importance des biens détenus varie peu (de 4 à 9 ha), hormis le cas d'un jeune cadet "paysan sans terre", dont la quasi totalité de la SAU est en location. La superficie de la "propriété " moyenne a peu varié (environ 6 ha). L'exiguïté dés parcelles, encore lisible dans le paysage ( 25 ares en moyenne), et lcur grande dispersion témoignent de l'ancienne pression démographique et de la volonté d'utiliser les différents terroirs, mais aussi de l'attachement à la terre qui a bloqué toute possibilité de remembrement.

Dans ce contexte, il n'y a eu aucun mouvement de concentration des exploitations. Les structures foncières (en propriété) sont restées d'une grande stabilité; ainsi la diversification des exploitants au cours des dix denières années s'est-elle essentiellement opérée sur des données sociales, fonctionnelles et culturelles : diversité des projets professionnels, des modes de vie, des sources de revenus, des statuts familiaux, des perspectives de succession (tabl. 2).

b. La diversification des propriétaires fonciers s'accompagne d'un blocage du marché foncier agricole. Depuis 1954, date avant laquelle nous ne disposons que de données fragmentaires ou peu fiables, la mobilité foncière n'intéresse que 17 ha ( $8 \%$ de la SAU). Dix hectares ont changé de propriétaire agricole et sept ont été vendus en terrain à bâtir. Sur les 10 hectares, 4 seulement ont été achetés ; cela concerne 8 agriculteurs pour une superficie moyenne de 0,53 ha par parcelle; les 6 autres hectares ont changé de propriétaires à l'occasion d'héritages. Les 7 hectares vendus en terrain à bâtir représentent une vingtaine de transactions portant sur de petites parcelles. Elles attestent d'une pénétration non négligeable d'intérêts non agricoles porteurs de mutation dans les fonctions du territoire donc de spécu- 
lations liées au développement du tourisme (Menjon et Sartor, 1972). La multiplication des résidences secondaires au cours des dix dernières annćes (22 en 1984, donc autant que de résidences permanentes) mite les territoires de fonds de vallée et constitue un danger pour l'agriculture en l'absence de plan d'occupation des sols. Le refus de définir un zonage du territoire traduit, une fois encore, l'absence de choix qui engage l'avenir de cette collectivité locale. Les plus grandes inquićtudes des agriculteurs portent cependant sur les 91 ha qui appartiennent à des proprićtaires, non agriculteurs, auxquels vont venir s'ajouter les 28 ha détenus par des retraités sans successeurs qui maintiennent encore une petite activité agricole. La fuite du potentiel de l'agriculture est beaucoup plus importante que ne le laisse supposer la seule analyse du marché foncier (Barrué-Pastor et :l.. 1981). En effet, c'est sur les mécanismes de la transmission patrimoniale que repose encore l'essentiel de la reproduction ćconomique et sociale.

\section{L'effet des coutumes successorales.}

L'histoire des sociétés pyrénéennes est fortement marquée par les pratiques coutumières relatives au mode de succession patrimoniale. Elles ont régi jusqu'à 1930 environ, et imprégnent ercore, les relations familia!es et sociales par une maîtrise des conditions d'appropriation des biens, donc de leur gestion. La soumission totale à l'autorité du chef de famille, qui est l'une des deux dispositions fondamentales ayant force de loi avec la transmission intégrale à l'aîné des garçons, se traduit aujourd'hui encore dans le rythme très lent de passation cles pouvoirs.

A Oô ce n'est qu'après le décès des deux parents que le successeur accède à la fois à la propriété et à la succession réelle de l'exploitation (nous n'avons enregistré qu'un cas de succession réglé du vivant au cours des trente dernières années). En 1980, cinq des exploitants (ayant entre 32 et 50 ans) ne sont pas encore chefs d'exploitation (un cas de statut d'aide-familial à 60 ans). Tant que les prédécesseurs sont en vie, l'héritier successeur n'a aucun pouvoir de décision sur l'exploitation, puisqu'il n'en est pas le propriétaire. Cette coutume est un frein au développement des exploitations et un facteur d'accélération de la disparition des plus petites. Cependant, les biens fonciers des exp!oitations éliminées ne viennent pas pour autant renforcer ceux des exploitations en activité. Pour que les patrimoines ainsi libérés viennent alimenter le marché foncier local, il faudrait que les successions soient réglées. Or, depuis la seconde guerre mondiale on observe l'expansion rapide de la pratique de l'indivision (Barrué-Pastor et al., 1982). Sur les 30 exploitations recensées en 1954, 13 étaient en indivision (43\% des exploitations). En 1980, un quart de siècle après, deux cas seulement ont été réglés, cinq ont disparu, et six cas d'indivision se maintiennent, alors qu'il ne reste plus que 16 exploitations en activité. 
Si l'indivision est un blocage pour le développement de l'élevage, elle l'est aussi pour le développement de l'urbanisation et du tourisme. A ce titre, elle semble symboliser la contradiction entre les deux logiques qui s'aifrontent et qui pénétrent les mécanismes traditionnels de régulation, dans la famille et la collectivité. La première se réfère à la capacité de résistance des agriculteurs, qui ont trouvé là un moyen de s'opposer à l'application du Code civil qui prône le partage entre tous les héritiers et donc la destructuration des entités famille-exploitation. La seconde raison se réfère à un mécanisme qui lui est opposé et qui découle des possibilités récentes de spéculations liées à la mutation de fonction possible des terres sous l'effet du tourisme. Les cohéritiers non agriculteurs ont eu tendance à laisser le domaine foncier indivis, non pas comme autrefois pour éviter le morcellement des biens, mais pour éviter les déconvenues d'un zonage tardif sur des attributions trop précises, notamment dans les zones sans plan d'utilisation des sols (Wisner, 1979).

Quoi au'il en soit, la possibilité de transformer une terre agricole en terrain à bâtir a définitivement détruit le système successoral coutumier basé sur une logique unique de préservation de la maisonnée. En cela elle a accéléré le dépérissement de l'agriculture de montagne et détruit, après avoir longtemps été son outil de sauvegarde, sa légitimité sociale et la marginalisation des éleveurs dans la société locale. Les effets de cette dévalorisation sociale et culturelle ne sont pas négligeables sur le terrain économique et écologique de la régression agricole. Ils ont eu un effet déterminant, notamment sur la génération qui a aujourd'hui entre 50 et 60 ans et qui se définit comme la génération des «sacrifiés». Dans nombre de familles, l'aîné a cédé son droit de succession sur l'exploitation à celui qui a bien vculu "rester à la terre ", mais sans céder son droit au patrimoine. Dans un contexte où le statut d'agriculteur n'est souvent obtenu qu'à l'âge de la retraite, l'insécurité et l'incertitude du lendemain sont venues accroître le désengagement de ces éleveurs. Travaillant des terres sur lesquelles ils n'ont ni assurance d'en hériter, ni celle d'en avoir une jouissance durable, incertains de bénéficier des fruits d'un travail d'entretien à long terme, privés de la possibilité de prendre des responsabilités, donc des initiatives, ces exploitants ont manifesté une attitude "passive» à un moment où l'industrialisation se développait et où l'intégration de l'agriculture à l'économie de marché devenait totale.

\section{Conditions d'accès des exploitants agricoles à l'usage du foncier.}

Dans ce contexte où les agriculteurs perdent progressivement la maîtrise de la propriété foncière agricole, les stratégies déployées pour obtenir l'usage des terres nécessaires aux exploitants s'exercent surtout par le biais de la location des terres libérées. Aujourd'hui, les surfaces en propriété ( 6 ha en moyenne) ne sont pas totalement utilisées (de 1 à 6 hectares selon les exploitations) et la part des 
terres utilisées en location varie de 10 à $95 \%$. Tous les éleveurs ont fondé leur fonctionnement sur des stratégies locatives; pour les plus âgés elle repose sur la recherche de commodités (proximité, facilité du travail, etc.; pour les exploitants en pleine activité, c'est une nécessité, compte tenu de l'augmentation nécessaire des effectifs des troupcaux pour assurer un maintien des revenus (plus de $35 \%$ en 10 ans). S'il est aujourd'hui assez facile d'obtenir l'usage des prés, encore faut-il en connaître les conditions. A Oô, comme dans tous les villages de la vallée, il est presque impossible d'obtenir des locations avec des baux écrits. L'indemnité volontaire de départ n'a pas eu d'effet incitatif. Sur les 43 hectares de SAU utilisés en location, 37 le sont par location verbale non déclaréc, 6 seulement font l'objet de baux écrits au bénéfice de deux jeunes installés au prix d'efforts de plusielirs années. Le paiement des loyers passe par des échanges en nature ou en service, rarement par des moyens financiers. Les concurrences entre les différents actifs agricoles alimentent les conflits locaux et aggravent la précarité de l'accès au foncier par une rotation importante des bénéficiaires. Certains jeunes exploitants estiment qu'un tiers des terres louées change chaque année de locataire. Ainsi la diminution du nombre des exploitants et des actifs agricoles n'est-elle pas suivie d'une concentration foncière des exploitations. La redistribution de l'usage des terres se fait dans des conditions telles qu'elle est devenue un élément moteur de la déprise tant agronomique que sociale. Ces locations ont permis d'augmenter la part des prés de fauche dans la SAU utilisée par chacun, d'utiliser préférentiellement les meilleures parcelles : abandon des parcelles en propriété mal situées, et location de parcelles plus favorables.

La précarité des conditions de location met l'élevage en péril; elle aggrave l'appauvrissement des prés de fauche, car les agriculteurs hésitent à engager des travaux d'entretien et des frais de fertilisation sur des parcelles dont ils ne sont pas sûrs de jouir longtemps. Elle compromet les chances de bénéficier de la « dotation jeunes agriculteurs" pour l'installation, car il n'y a aucune possibilité d'obtenir sur place les 14 hectares de la "surface minimum d'installation ": pour s'installer, les jeunes sont obligés d'aller chercher dans les vallées voisines les baux écrits que leur impose l'administration. Ils sont ainsi assurés de la jouissance de biens qu'ils n'utiliseront jamais alors que tout leur fonctionnement repose sur des terres exploitées dans la plus totale incertitude.

\section{Perspectives d'avenir et possibilités d'intervention}

Parler de développement en montagne et s'attaquer au processus régressif de l'agriculture, c'est créer les possibilités d'installation réelle et mettre en place les éléments d'une gestion, de l'usage et du renouvellement des ressources des terres agricoles. Ces éléments relèvent à la fois de la configuration locale des rapports sociaux et 
de leurs rapports au territoire et des logiques institutionnelles de l'Etat. L'histoire et l'évolution récente du système agro-pastoral de la vallée étudiée attestent une interpénétration des facteurs écologiques et sociologiques, dont les effets se cumulent et accélèrent le processus régressif de la déprise agricole. L'analyse des rapports entre les pratiques pastorales choisies par une société et le devenir de son territoire n'a de sens que resituée dans la dynamique du changement économique et social.

\section{L'épuisement de la rente de situation compromet le renouvellement des ressources agro-écologiques.}

La comparaison des bilans minéraux et des caractéristiques botaniques des parcelles (Balent et Duru, 1984), a permis de mettre au point un modèle statistique rendant compte de l'évolution passée et de l'état actuel des surfaces pastorales. Il permet également de prévoir leur rythme d'évolution dans l'hypothèse où les modalités de leur mise en valeur actuelle (fertilisation, pâturage) demeurent inchangées. Grâce à ce modèle, nous avons montré que la notion de rente de situation est indissociable de la notion de flux. En effet, en l'absence de fertilisation, les parcelles évoluent d'un état initial (champs cultivé) à un état final, (pelouse oligotrophe ou friche ligneuse) en passant par un stade "optimal" de production et d'utilisation. Ce processus a débuté dans les années 1930. En 1925, en effet, toute la surface cadastrée était soit fauchée, soit cultivée (190 ha); dix ans plus tard, il y avait 7 ha uniquement pâturés, 42 ha en 1955 et 105 ha en 1980 (55\% d la SAL). Cette évolution très rapide s'est faite au détriment des champs qui sont passés dans le même intervalle de 92 ha à 10 ha. Si on prolonge la courbe d'évolution des champs pour les années à venir (fig. 5b) en supposant que les tendances d'évolution demeurent inchangées, les dernières parcelles cultivées auront disparu d'ici la fin du siècle et avec elle la rente de situation. En fait tout se passe comme si, au cours des soixante dernières années, les agriculteurs avaient mis en place un système de "pillage organisé et rationnel "d'un capital de fertilité, sans se soucier de sa reproductibilité. Nous retrouvons là les traces du profil sociologique des exploitants sans successeur et sans avenir. Ce cycle fonctionne tant que ses acteurs acceptent l'idée que la date d'épuisement de ce système correspond à la date d'arrêt de leur activité. Or, ce qui est nouveau, et qui vient totalement bouleverser cette logique de gestion, c'est l'installation de plusieurs jeunes agriculteurs. Cela signifie une augmentation du nombre d'animaux hivernés, qui nécessite un renversement de la dynamique régressive découlant de la gestion actuelle.

\section{La diversification et la marginalisation des éleveurs compromet leur capacité de résistance.}

Les exploitants actifs sont freinés dans leurs objectifs économiques, alors qu'ils sont, par ailleurs, marginalisés par rapport aux modèles 
de dévcloppement dominants. Après avoir été un facteur de préservation, les dernières traces des logiques coutumières ont accéléré la régression agricole. La pratique de l'indivision et le refus de porter altsinte au patrimoine ont bloqué non sculement le marché foncier et l'évolution possible des exploitations, mais encore toute possibilité d'échange et de fermage déclaré. Les locations verbales se développent et freinent l'entretien des terres agricoles par leur grande précarité. Le rôle des propriétaires non agricoles est plus grand que la proportion des terres qu'ils détiennent. Ils influent sur leur usage. Par le refus des baux écrits, ils agravent la pratique de la "cueillette " et la chute de la fertilité, par leur inertie, ils bloquent les possibilités d'amélicration des pâturages collectifs.

La structuration économique des rapports sociaux locaux s'est transformée, notamment sur la base des mutations de fonction possibles des territoires sous le poids du tourisme. En l'absence de zonag̣e clairement défini (P.O.S., par exemple) le tourisme impose une forte concurrence. Les prix ne cessent d'augmenter et tout proprićtaire à l'espoir de vendre au prix du terrain à bâtir. Les meilleurs terrains à construire sont, par ailleurs, les meilleures terres agricoles. Les résidences secondaires vont "miter" tout le fond de la vallée et modifier la circulation des troupeaux et les conditions de gestion des pâturages. Minoritaires, les propriétaires agriculteurs ont beaucoup de difficultés à préserver leur existence, liéc au contrôle des terres agricoles. L'exode agricole et l'arrivée de nouveaux résidents ont modifié les groupes sociaux locaux et les rapports de force. Dans ce contexte, les éleveurs actifs ne dominent plus le pouvoir municipal. Ainsi toutes les modifications de la conjoncture économique et sociale conduisent-elles à l'éclatement, sous le poids d'intérêts contradictoires, d'une cohésion villageoise centrée sur une agriculture active.

Les familles agricoles elles-mêmes sont atteintes. Non seulement elles participent à la spéculation liées aux mutations de fonction des territoires mais elles contribuent à l'atomisation sociale en projets individuels. Insćrés dans la logique du droit de la proprićté privée et de l'individualisme qui s'y rattache, victimes de la concurrence pour l'utilisation des prés et de l'obsolescence des pratiques collectives, les éleveurs ont beaucoup de difficultés à dépasser leur insécurité et leur divisions internes pour raisonner collectivement les pratiques pastorales. Par ailleurs, le mouvement social qui va dans le sens de l'hétćrogénéisation des statuts et des pratiques, révèle de nouvelles contradictions internes à l'agriculture. $\mathrm{Ce}$ climat social conflictuel bloque à la fois toute révision des règlements collectifs et la mise en place d'un nouveau mode de mise en valeur individuel.

Or dans la situation économique actuelle, où de plus en plus de jeunes ont fait le pari de s'installer dans l'agriculture, le problème de l'augmentation de la productivité du travail se pose de manière vitale. L'augmentation des effectifs des troupeaux ayant atteint bien souvent les limites structurelles des exploitations, elle se pose en 
termes d'intensification. Dans ce contexte, où propriété individuelle et propriété collective sont interdépendantes, l'élevage ne peut se maintenir ou se développer que si le consensus l'emporte sur la division sociale, l'intérêt d'un collectif sur l'individualisme.

\section{Le rôle de l'Etat.}

Si le devenir des éleveurs est en grande partie lié à leur capacité à se mobiliser sur un projet, un tel enjeu ne peut pas relever que de dispositions locales : il relève directement de l'action de l'Etat et des possibilités offertes par la législation française.

Au cours de la première grande période d'application du Code civil, une résistance s'est mise en place pour préserver l'essentiel des logiques coutumières sur lesquelles reposaient une certaine cohésion villageoise. A partir des années 1960, les perspectives nouvelles de l'économie montagnarde (tourisme notamment) et les dispositions institutionnelles qui s'y rattachent (Assier-Andrieu, 1981) ont favorisé le développement de la logique de la propriété privée et des partages prévus par la loi nationale. La possibilité d'affecter des parcelles de terres à la construction immobilière et d'augmenter considérablement leur valeur a définitivement rompu le sens des dernières pratiques coutumières de transmission des patrimoines. Il y a eu rupture du concensus successorial basé sur la reproduction de la «maison».

La destruction de cette logique institutionnelle familiale de l'unité agricole est arrivée à un moment marqué par l'absence de tout projet global d'aménagement du territoire de la part de l'Etat. Malgré le premier effort dans ce sens que représente la loi de 1985 sur la montagne, ses dispositions restent limitées et incapables d'inverser la tendance du processus de régression tel que nous l'analysons. Et ce, qu'il s'agisse de l'élargissement du pouvoir des SAFER, d'un assouplissement des conditions d'application de la loi sur la récupération des terres incultes, ou de l'extension des associations pastorales aux zones boisées. Quant à la procédure de recensement des terres en cultures, prévue à l'article 40 du Code rural, elle reste illusoire (Comby, 1984). Le flou de certaines dispositions est sans doute lié au déliquat équilibre que cette loi tente d'instaurer entre la protection des sites et le développement des activités.

\section{Possibilités d'intervention.}

Malgré ces lacunes les solutions techniques existent, mais elles doivent être raisonnées en termes de coût (individuel et social) et, d'autre part, en relation avec l'ensemble des interventions nécessaires à leur impact réel. Des expériences de débroussaillage, de désherbage, d'amendement par hélicoptère ont été faites, mais sont restées ponctuelles et d'un coût disproportionné avec l'amélioration des revenus procurés à l'élevage. A Oô, comme dans les autres communes du 
canton, les travaux d'amélioration réalisés s'inscrivent dans les programmes classiques des aides attribuées aux groupements pastoraux : il s'agit surtout de la pose de clôture en estives, du captage de points d'eau, de l'amélioration des cabanes des gardiens et des chemins d'accès. Dans la mesure où tous ces travaux ne s'inscrivent pas dans un programme qui porte sur l'ensemble des zones pastorales participant au calendrier de pâturage des troupeaux, ils relèvent d'un " saupoudrage » financier aux effets limités.

S'attaquer localement au processus régressif qui affecte l'élevage suppose de traiter conjointement le double problème de la reproductibilité agronomique et sociale. Le premier nécessite de poser simultanément les problèmes de gardiennage et de fertilité. Le second, suppose l'existence d'un groupe d'agriculteurs constituant un potentiel de résistance en faveur de la vie agricole. Ce dernier impératif se heurte à deux obstacles fondamentaux issus de la chute de la dynamique agricole: l'hétérogénéité des exploitants et l'importance des propriétaires non agricoles. D'où la nécessité de dépasser l'obstacle premier de l'hétérogénéité et d'obtenir sur un projet commun, accord minimum suffisant, mais nécessaire, pour affronter le blocage des propriétaires fonciers non agricoles. En effet, la réussite des actions engagées dépend de l'assurance qu'auront les éleveurs de profiter de leurs effets à moyen et long termes. Ces exigences ne peuvent se traiter sans remettre à l'ordre du jour la question de la dissociation du droit de propriété et du droit d'usage de la terre agricole. Cependant si le foncier se trouve à la base de tout problème d'aménagement, le poser au départ comme condition de développement semble vouer à l'échec toute perspective d'intervention, compte tenu des limites fournies par l'appareil législatif dans ce domaine.

Sur ces bases, une opération d'amélioration pastorale des pâturages de la commune d'Oô (12) a été lancée. Elle tente de contourner le problème crucial du foncier et de tester la possibilité d'un usage collectif de surfaces encore de statut privé. Elle se propose aussi de réorienter les fonds publics d'aménagements pastoraux d'altitude vers les surfaces de fonds de vallée. Trois séries d'actions sont envisagées : amélioration de la répartition des charges animales, maintien et rétablissement de la fertilité du sol, restauration et entretien des terroirs de soulane.

Ce projet, conçu avec les agriculteurs et avec la municipalité de la commune d'Oô - garante de l'accord des propriétaires fonciers -, essaie d'évaluer, malgré la contrainte foncière, les possibilités d'une mise en œuvre d'ensemble avec une participation des agriculteurs actifs. La mobilisation des agriculteurs dans la réflexion sur des

(12) Programme de recherche-développement «Maintien d'éleveurs et développement de l'élevage dans les Pyrénées Centrales " financé par la commission agronomique de la CEE au titre des opérations pilotes. (Groupe de recherches INRA - URSAD, CIMA (LA 366), CNRS, ITOVIC Toulouse, 1983-1985). 
questions qui intéressent l'évolution globale du système pastoral sur l'ensemble du territoire communal montre qu'une redynamisation de la vie communautaire reste possible. Elle est non seulement susceptible de proposer des réponses efficaces à la question de la reproductibilité des ressources pastorales mais encore de tester des moyens de contourner la difficile question foncière qui reste du ressort de la législation nationale.

\section{Conclusion}

Entraînés dans une dynamique défavorable au développement de l'agriculture, les éleveurs des Pyrénées centrales accentuent par leurs pratiques le mouvement de déprise et d'embroussaillement. Autrefois strictement contrôlées par la communauté, les règles de gestion collective de l'espace pastoral reflètent toujours une survivance des logiques antérieures mais viennent, de plus en plus, d'initiatives individuelles. Le processus de destruction d'une cohésion fondée sur l'agriculture s'est accéléré sous le poids des conflits d'intérêt, de la domination du droit de propriété et des interventions de l'Etat. La modification des conditions d'utilisation de l'espace est pour partie inscrite dans le paysage pastoral : enfrichement, embroussaillement, hydromorphie des sols, érosion (photo. 10 et 11). Par contre, la baisse de la fertilité des sols et la mauvaise répartition des charges animales sont beaucoup plus difficile à percevoir par les agriculteurs alors qu'elles compromettent gravement la reproductibiiité des ressources pastorales.

Pour mettre en évidence les mécanismes qui commandent ces processus nous avons rassemblé des données écologiques et socioéconomiques à différentes échelles (parcelle, quartier de pâturage, territoire communal) et sur différents pas de temps (journée, saison de pâturage, année). Après avoir mis en perspective historique ces différents éléments, nous avons pu proposer un système explicatif et prédictif des mécanismes régressifs en fonction des rythmes identifiés, au niveau agronomique notamment (fig. 12).

Les problèmes de transmission du patrimoine (logiques familiales), de la spéculation liée au tourisme et l'absence de garanties dans les locations de parcelles (logique socio-économique) sont autant d'obstacles à une bonne gestion du potentiel de production des surfaces fourragères (logique agro-écologique). Les stocks fourragers nécessaires à l'hivernage d'un grand nombre d'animaux sont compromis. Or le maintien d'un effectif important d'animaux pendant l'hiver conditionne l'accès à des revenus décents et le maintien d'un nombre suffisant d'éleveurs pour assurer la permanence d'un tissu social.

La question de la double reproduction agronomique et sociale, telle que nous l'avons posée, apparaît dominée par celle des rapports de force. L'étude du problème foncier met en évidence que le pouvoir 


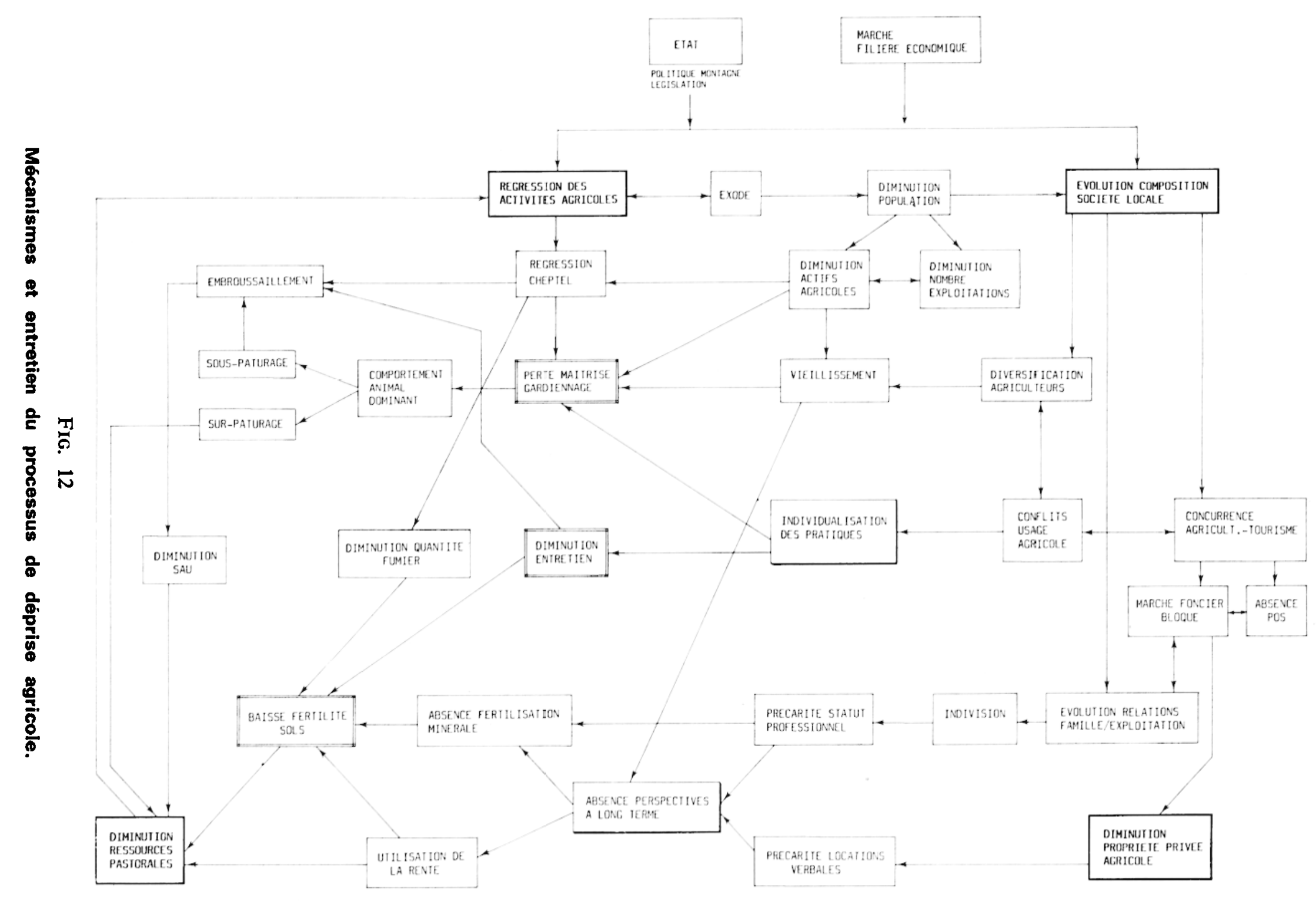


de surmonter le processus de déprise est lié surtout à la propriété du sol. L'appropriation foncière obéit à un double mécanisme : celui de l'évolution de la législation (donc de la société française) et des pratiques spécifiques enracinées dans les us et coutumes locaux.

En partant de problèmes de la pratique sociale, on a confronté les connaissances acquises aux possibilités d'action de développement. La démarche interdisciplinaire confère à cette tentative une globalité capable de saisir la réalité de phénomènes complexes; elle devient alors, en elle-même,une force de proposition qui intègre la diversité des éléments concernés et remet en cause et les pratiques du développement et, plus globalement, les pratiques sociales jusqu'à leurs incidences techniques permettant d'apprécier les limites d'interventions partielles sur le processus de déprise.

Dans quelle mesure pourraît-il y avoir un autre mode d'organisation des échanges et d'utilisation des ressources du milieu ? Les pouvoirs publics souhaitent reconstituer une certaine rentabilité des territoires montagnards.

C'est la situation définie par Jollivet (1978) : «Il s'agit en somme d'inventer un système qui permette la réalisation d'une nouvelle rente foncière ". La monétarisation des opérations de production ou de reproduction sociale ou biologique est une nécessité croissante, par le recours au marché ou l'attribution de prestations diverses en argent. Si la loi sur la montagne reconnait à l'agriculteur le premier rôle dans le développement rural, elle met en doute la rentabilité économique et suggère une mutation (partielle) de fonction de l'élevage en rapport avec la protection des paysages montagnards; dans ce contexte, il aurait un rôle d'entretien et deviendrait une activité de service chargée de limiter le coût social de la désertification. Mais même dans cette hypothèse où la logique économique se fonderait sur "la production paysagère", la gestion actuelle des territoires pastoraux ne suffirait pas au maintien des équilibres écologiques. Il semble difficile de demander aux derniers éleveurs montagnards d'assumer seuls le poids d'un héritage. 


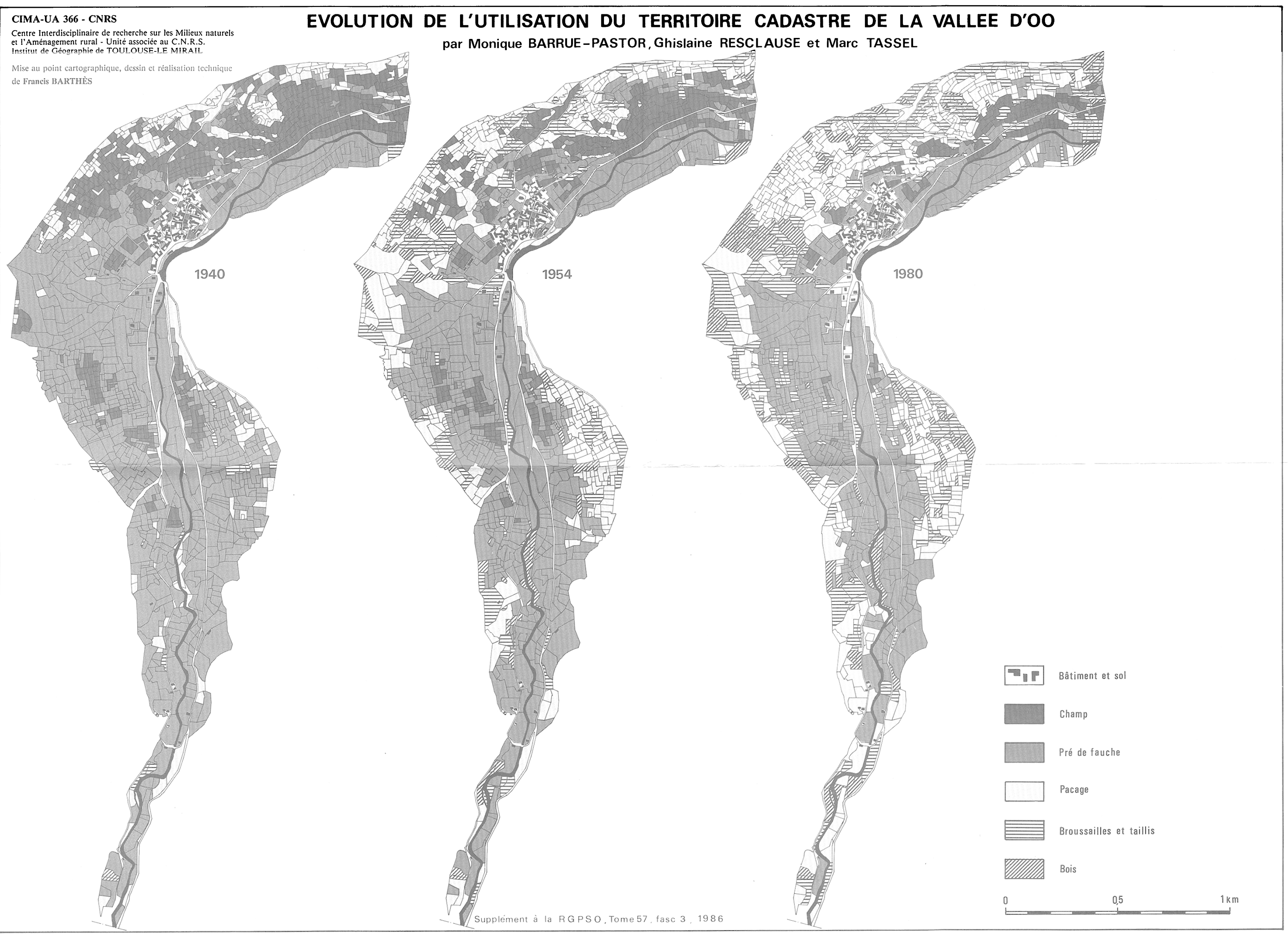




\section{Bibliographie}

ARNol.D G.W., DudziNski M.L., 1978. Ethology of free-ranging domestic animals. (Developments in animal and veterinary sciences 2), Amsterdam Elsevier.

Assier-A.Noriet L., 1981. Coutume et rapports sociaux. Paris, CNRS.

BALENT G., 1984. Influence of grazing on the botanical composition of previously cultivated fields. The example of the Pyrenees. Second International Rangeland Congress, May 1984, Adelaide, Australia, (sous presse).

Bali:NT G., Charpenteau J.L., 1984. The importance for grazing research of a model for calculating the spatial distribution of grazing pressure based on the observation of behaviour. Second International Rangeland Congress, 1984, Adelaide, Australia, (sous presse).

BALFN G., Derv M., 1984. Influences des modes d'exploitation sur l'évolution des surfaces pastorales: cas des Pyrénées Centrales. Agronomie, 4 (2).

Balint G., GiboN A., 1980. Le pâturage hivernal dans les Pyrénées Centrales. Recherches sur l'élevage en Pyrénées. Toulouse, INRA.

Balint G., GiboN A. 1986. Activité alimentaire des brebis sur les pâturages naturels des Pyrénées pendant la période hivernale. Journées des recherches sur l'alimentation et la nutrition des herbivores, INA Paris-Grignon: 21-22 mars 1985, Reprod. Nutr. Dévelop., 26 (1 B).

BARRIÉ:-PASTOR, 1980. Utilisation du territoire, marché foncier et système social. Recherches sur l'élevage en Pyrénées. Toulouse, INRA.

BARRLÉ-PASTOR, 1980. Contribution socio-économique au rapport scientifique: Recherches pluridisciplinaires sur les problèmes de l'élevage pyrénéen. (multigr.).

Barrúf-Pastor M., 1983. Paroles d'éleveurs pyrénéens... ou le développement des zone difficiles en question, Nouvelles Campagnes, 25.

Barkué-Pastor M., Bages R., Blanc R., Broueilh M., 1981. Spécificité des marchés fonciers locaux et stratégie des agriculteurs. Economie rurale, 142.

Barrué-Pastor M., Rieu-Gout A., Tassel M., 1982. Stratégies familiales de transmission du patrimoine et dynamique agricole dans les Pyrénées. Colloque national A.R.F., Tours.

Barrué-Pastor M., Resclause G., Tassel M., 1986. Atlas foncier pyrénéen (à paraître).

BAzIN G., 1983. Processus de régression et crises des agricultures montagnardes. INRA, (multigr.).

Bazin G., Larrère G.-R., De Montard F.-X., Lafarge M., Loiseau P., 1983. Système agraire et pratiques paysannes dans les Monts-Dômes. Paris, INRA.

Bertrand G., 1975. Pour une histoire écologique de la France rurale. Hisloire de la France rurale (sous la direction de G. DuBY et A. Wallon, Paris, Seuil, t. 1. 
Brun A., Chassany J.-P., Naert B., Osty P.-L., Petit F., 1978. Le Cause Méjan. Paris, INRA.

Chevalier M., 1980. La vie humaine dans les Pyrénées Ariégeoises. Tarascon-surAriège, Résonnances.

Coмby J., 1984. Protéger et/ou ménager la Montagne. Etudes Foncières, 23.

Despres Ph., Soulas C., 1981. Contribution à l'étude de l'élevage pyrénéen : 2. Le pâturage hivernal. (Mémoire de fin d'étude ENSA Montpellier). Toulouse, INRA - URSAD.

Duru M., Balent G., 1985. Fertilisation dans les exploitations d'élevage des Pyrénées Centrales. Pratiques des éleveurs. Références expérimentales. Deuxième forum national de la fertilisation raisonnée, (Atelier Elevage et Prairics permanentes) Toulouse, janvier 1985.

DUNOR M.-C., 1979. Utilisation et gestion collectives de l'espace agricole dans les Pyrénées Centrales. (Maîtrise de géographie), Université de Toulouse-Le Mirail.

FERAL P.-L., 1981. L'application des lois de 1860,1864 et 1882 sur le reboisement et le gazonnement des Montagnes: Les fruitières du Haut-Comminges, Revue du Commniges, 94 (1).

Fremont A., Chevalier J., Herin R., Renaud J., 1984. Géographie sociale, Paris, Masson.

GrBon A., 1981. Pratiques d'éleveurs et résultats d'élevage dans les Pyrénées Centrales. (Thèse Ingénieur Docteur) INA Paris-Grignon.

GrafF B., 1979. L'Oueil et le Larboust, 1900-1950. Deux vallées paysannes (Etude des systèmes de production). (Thèse de $3^{\mathbf{e}}$ cycle de Géographie), Université de Toulouse-Le Mirail.

GROUPE DE RECHERCHES INRA-ENSAA, 1977. Pays, paysans, paysages dans les Vosges du Sud. Les pratiques agricoles et la transformation de l'espace. Paris, INRA.

Higounet Ch., 1949. Le Comté de Comminges de ses origines à son annexion à la couronne. Toulouse-Paris, Privat-Didier.

INRA, 1983. La Margeride : la montagne, les hommes. Paris, INRA.

JOLLIVET M., 1978. Rente foncière et gestion des ressources naturelles. Etudes rurales, $71-72$.

Journal d'Agriculture Pratique, Société d'Agriculture de la Haute-Garonne, Conférences agricoles des 23-24-25 mai 1861.

LARRÈre R., 1984. Processus de régression et crises des agricultures, montagnardes. INRA, (multigr.).

Lorseau P., 1983. Histoire de la mise en valeur, écologie et perspectives d'utilisation pastorale des zones marginales. Système agraires et pratiques paysannes dans les Monts-Dômes, Paris, INRA.

MENJON L., SARTOR A., 1972. La vie rurale et le tourisme dans les vallées d'Oueil et du Larboust. Maîtrise de géographie, Université de Toulouse-Le Mirail.

Montard F.-X. DE, Balent G., Chapot J.-Y., Duru M., Jeannin B., Lafarge M., LanGlet A., MEtTauer H., 1981. Potentialités agroclimatiques et productivité agricole. L'INRA et la Montagne.

MORINIERE J.-L., 1984. Le Louron. Une vallée pyrénéenne au passé harmonieux, Toulouse.

ParaIN C., 1971. Fondements d'une ethnologie historique de l'Aubrac. L'Aubrac, (tome II); Paris, CNRS.

Pernet G., Lenclud D., 1977. Berger en Corse. Essai sur la question pastorale. Grenoble, Presses universitaires.

RaWsKI C., 1983. Contribution à l'étude de l'élevage pyrénéen. Etude de l'utilisation des surfaces pastorales par les troupeaux: Modélisation et informatisation des circuits de pâturage dans la vallée d'Oô. (Mémoire de fin d'étude, ENSA, Montpellier), INRA-URSAD, Toulouse. 
R.C.P. Aubrac, 1973-1974. Etude ethnologique, linguistique, agronomique et économique d'un établissement humain. Paris, CNRS.

Soli.fT J.-F., 1981. Aspects de la société pyrénéenne au XIX" siècle : le cas des vallées d'Oueil et du Larboust. Revue du Comminges, 94 (1).

WiBER E., 1983. La fin des terroirs. Paris, Fayard/Editions Recherches.

WISNi:R C., 1979. Changement social en montagne: l'exemple de Ceillac (Queyras). Recherches Economiques et Sociales, 15. Paris, Documentation Française.

Rístarí. - Au cours du siècle écoulé, les activités agricoles en montagne ont connu une régression très importante. Dans les Pyrénées centrales, les strictes règlementations communautaires visant l'usage du territoire pastoral se sont relâchées. La diversification sociale des agriculteurs, le blocage du marché foncier, la diminution des troupeaux, et le développement de pratiques pastorales individuelles, ont entraîné une baisse générale de la qualité des parcours et de la fertilité des sols qui pose le problème de la reproductibilité des ressources pastorales. Après une analyse des processus de déprise, dans leurs déterminants agro-écologiques et sociaux, sont envisagées les possibilités d'améliorer la gestion du territoire pastoral et d'assurer la pérennité de ses ressources dans une perspective d'une relance des activités agricoles en montagne.

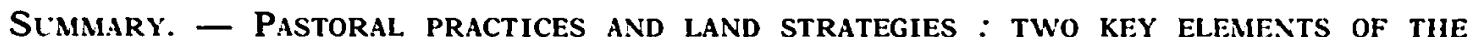
DECLINE OF MOUNTAIN IIUSBANDRY IN THE CENTRAL PYRENEES. During this century mountain agricultural activity has declined a lot. In the central Pyrénces strict village rules aimed at controlled pastoral uses have strongly decreased. The social diversification of farmers, the block on the land market, the drop in number of animals as well as the development of individual pastoral practices have procluced a drop in the quality of pasture and soil fertility which poses the problem of the reproduction of natural pastoral resources. After an analysis of the process of decline, and some speculation about agro-ecological and social factors, the authors stress the necessity of improving the management of such pastoral areas and their resources.

Resumen. - Pastoreo y estrategia de PRopiedad : Claves del Proceso de decadenCIA DE LA GANADERIA DE MONTAÑA. Durante el pasado siglo, las actividades agrícolas de montaña experimentaron un notable retroceso. En el Pirineo Central, las reglamentaciones comunitarias muy estrictas que tendian a regular el aprovechamiento de los pastizales perdieron eficacia. La diversificación social de los agricultores, el inmovilismo del mercado de la tierra, la disminución global de la cabaña así como el desarrollo de un pastoreo individual acarrearon una disminución de la calidad de los recorridos y de la fertilidad de los suelos, planteando la cuestión de la reposición de los recursos en pastos. Análisis del proceso actual de abandono con sus condicionantes agro-ecológicos y sociales. Perspectivas de mejora de la gestión de los pastizales y de conservación de sus cecursos dentro del marco de una reactivación de la agricultura de montaña.

Mots-Clés. - Pyrénées, Haut-Comminges, Vallée d'Oô, Elfvage ovin et bovin, pratiques pastorales, ressources fourragères, propriété fóncière, déprise agricole, SOCIÉTÉ RURAI.E, droit coutumier, reproductibilité, système social AGRO-ÉCOLOGIE,

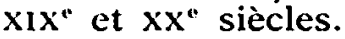

